\title{
A Weak Form of Amenability of Topological Semigroups and its Applications in Ergodic and Fixed Point Theories
}

\author{
Ali Jabbari ${ }^{1}$, Ali Ebadian ${ }^{2}$, and Madjid Eshaghi Gordji ${ }^{3}$
}

\begin{abstract}
In this paper, we introduce a weak form of amenability on topological semigroups that we call $\varphi$-amenability, where $\varphi$ is a character on a topological semigroup. Some basic properties of this new notion are obtained and by giving some examples, we show that this definition is weaker than the amenability of semigroups. As a noticeable result, for a topological semigroup $S$, it is shown that if $S$ is $\varphi$ amenable, then $S$ is amenable. Moreover, $\varphi$-ergodicity for a topological semigroup $S$ is introduced and it is proved that under some conditions on $S$ and a Banach space $X, \varphi$-amenability and $\varphi$-ergodicity of any antirepresntation defined by a right action $S$ on $X$, are equivalent. A relation between $\varphi$-amenability of topological semigroups and existance of a common fixed point is investigated and by this relation, HahnBanach property of topological semigroups in the sense of $\varphi$-amenability defined and studied.
\end{abstract}

\section{CONTEnts}

1. Introduction

2. $\varphi$-Amenability

3. Some Hereditary Properties 8

4. $\varphi$-Ergodic Properties 13

5. $\varphi$-Amenability and Fixed Point Property 17

\begin{tabular}{|l|l|}
\hline Acknowledgment & 23 \\
\hline
\end{tabular}

References 23

\section{Introduction}

Let $S$ be a semigroup. A character (semi-character) on $S$ is a nonzero map $\varphi$ : $S \longrightarrow \mathbb{T}(S \longrightarrow \mathbb{D})$ such that $\varphi(s t)=\varphi(s) \varphi(t)$, for all $s, t \in S$. We denote the space of characters (semi-characters) on $S$ by $\Delta_{S}(S)\left(\Phi_{S}\right)$ and $\Delta_{S}(S) \subset \Phi_{S}$.

2010 Mathematics Subject Classification. Primary: 43A07, 22D15, Secondary: 22A20.

Key words and phrases. Character, Character amenability, Ergodic theory, Fixed point property, Hahn-Banach property, semi-character, semigroup. 
For every $\varphi \in \Phi_{S}$, the map $\widehat{\varphi}: \ell^{1}(S) \longrightarrow \mathbb{C}$ defined by $\widehat{\varphi}(f)=\sum_{s \in S} \varphi(s) f(s)$ $\left(s \in S, f \in \ell^{1}(S)\right)$, is the character on $\ell^{1}(S)$, and indeed all of characters on $\ell^{1}(S)$ constructed by this method, see [6, 22 for more details.

Let ${ }_{\varphi} \mathcal{M}^{S}$ be the class of Banach $S$-bimodules such as $X$ for which the left module action of $S$ on $X$ is given by $s \cdot x=\varphi(s) x$, for all $s \in S$ and $x \in X$. Similarly, $\mathcal{M}_{\varphi}^{S}$ is the class of Banach $S$-bimodules $X$ for which the right module action of $S$ on $X$ is given by $x \cdot s=\varphi(s) x$, for all $s \in S$ and $x \in X$.

Let $C(S)$ be the Banach algebra of complex valued continuous bounded functions on $S$. We can consider $C(S)$ as a member of $\varphi_{\varphi} \mathcal{M}^{S}$ and $\mathcal{M}_{\varphi}^{S}$, because $\varphi$ is continuous. A function $f \in C(S)$ is called left uniformly continuous if $\lim _{\alpha}\left\|s_{\alpha} \cdot f-s \cdot f\right\|_{\infty}=$ 0 , whenever $s_{\alpha} \longrightarrow s$. We denote the Banach algebra of all right (left) uniformly continuous functions on $S$ by $R U C(S)(L U C(S))$. Let $E$ be a linear subspace of $C(S)$ which contains the constant function $1_{S}$. A mean on $E$ is a functional $m \in E^{*}$ such that $m\left(1_{S}\right)=\|m\|=1$. If $E$ is closed under module actions, then the mean $m$ is called left (right) invariant if $s \cdot m=m(m \cdot s=m)$, for all $s \in S$.

Let $S$ be a topological semigroup, for $s \in S$, the left translation $l_{s}$ of $C(S)$ by $s$ is defined by $l_{s} f\left(s^{\prime}\right)=f\left(s s^{\prime}\right)$, for all $f \in C(S)$ and $s^{\prime} \in S$ and the right one denoted by $r_{s}$ such that $r_{s} f\left(s^{\prime}\right)=f\left(s^{\prime} s\right)$.

A semigroup $S$ is called left (right) amenable if there is a left (right) invariant mean on $R U C(S)(L U C(S))$, i.e., there is a linear functional $m$ in $R U C(S)^{*}\left(L U C(S)^{*}\right)$ such that $m\left(l_{s} f\right)=m(f)\left(m\left(r_{s} f\right)=m(f)\right.$, for all $f \in R U C(S)(L U C(S))$ and $s \in S$. Moreover, $S$ is called amenable if it is both left and right amenable.

Let $S$ be a topological semigroup and let $X$ be a Banach $S$-bimodule. A bounded derivation is a weak*-continuous map $D: S \longrightarrow X^{*}$, such that $D(s t)=s \cdot D(t)+D(s) \cdot t$, for all $s, t \in S$, and $\sup _{s \in S}\|D(s)\|<\infty$. The bounded derivation $D$ is called principle, if there is an element $f \in X^{*}$ such that $D(s)=s \cdot f-f \cdot s=\operatorname{ad}_{f}(s)$, for all $s \in S$. If every derivation on semigroup $S$ is principle, then $S$ is called Johnson amenable. The Johnson amenability of semigroups and groups studied in [15.

The amenability of (topological) semigroups and topological groups have close connections with amenability of Banach algebras defined on semigroups and groups. A well-known result related to these connections, is the Johnson Theorem [11]: the locally compact group $G$ is amenable if and only if $L^{1}(G)$ is amenable. A Banach algebra $\mathfrak{A}$ is said to be amenable if, for any Banach $\mathfrak{A}$-bimodule $X$, every continuous derivation $D: \mathfrak{A} \longrightarrow X^{*}$ is inner.

Let $\mathfrak{A}$ be a Banach algebra and $\sigma(\mathfrak{A})$ is the carrier space of $\mathfrak{A}$, and $\varphi \in \sigma(\mathfrak{A})$ is a homomorphism from $\mathfrak{A}$ onto $\mathbb{C}$. Assume that $\varphi \in \sigma(\mathfrak{A}) \cup\{0\}$ and $X$ is an arbitrary Banach space, then $X$ can be viewed as Banach left or right $\mathfrak{A}$-module by the following actions

$$
a \cdot x=\varphi(a) x \quad \text { and } \quad x \cdot a=\varphi(a) x \quad(a \in \mathfrak{A}, x \in X) .
$$

The Banach algebra $\mathfrak{A}$ is said to be left character amenable (LCA), if for all $\varphi \in$ $\sigma(\mathfrak{A}) \cup\{0\}$ and Banach $\mathfrak{A}$-bimodules such as $X$ for which the left module action is given by $a \cdot x=\varphi(a) x(a \in \mathfrak{A}, x \in X)$, every continuous derivation $D: \mathfrak{A} \longrightarrow X^{*}$ is inner. Right character amenability (RCA) is defined similarly by considering Banach 
A-bimodules such as $X$ for which the left module action is given by $x \cdot a=\varphi(a) x$, and $\mathfrak{A}$ is called character amenable (CA) if it is both left and right character amenable. The notion of character amenability of Banach algebras was defined by Sangani Monfared in 17] and the concept of $\varphi$-amenability of Banach algebras introduced by Kaniuth and et al. in 12 .

Let $S$ be a topological semigroup and $0 \neq \varphi \in \Delta_{S}(S)$. This paper, considers the concept of left (right) $\varphi$-amenability of topological semigroup $S$ and some notions that have connections with it.

In section 2 , we introduce left (right) $\varphi$-amenability of topological semigroups and show that this new notion is different from amenability of semigroups (groups). Moreover, some results about relations between $\varphi$-amenability of semigroups (groups) and character amenability of semigroup algebras (group algebras) are obtained.

In section 3 , we considers some hereditary properties of $\varphi$-amenability and define the strongly left (right) $\varphi$-amenability on subsemigroups of semigroups where with this definition, we show that strongly left (right) $\varphi$-amenability of a left (right) thick subsemigroup implies that the left (right) $\varphi$-amenability of a semigroup and vice versa.

Section 4 deals with left $\varphi$-egodicity that we introduce it in that section and investigate some relations between left $\varphi$-amenability of $S$ and left $\varphi$-egodicity. Moreover, we obtain a characterization of $\varphi$-amenability of $S$ in terms of antirepresentations of $S$ on a Banach space.

Finally, in section 5, we study of the existence of a common Fixed point in compact convex sets that $S$ has continuous affine actions on. Moreover, we define Hahn-Banach Property related to $0 \neq \varphi \in \Delta_{S}(S)$ and as an interesting result, we characterize $\varphi$ amenability of topological semigroups.

\section{2. $\varphi$-Amenability}

Let $S$ be a topological semigroup and $\varphi \in \Delta_{S}(S)$. In this section, we study left (right) $\varphi$-amenability of the semigroup $S$ and obtain some necessary and sufficient related to left (right) $\varphi$-amenability of $S$ such as Theorem 2.12. We start this section with the new definition as follows

Definition 2.1. Let $S$ be a topological semigroup and $0 \neq \varphi \in \Delta_{S}(S)$. We say that

(i) $S$ is left $\varphi$-amenable if, for each Banach $S$-bimodule $X \in_{\varphi} \mathcal{M}^{S}$, every bounded derivation $D: S \longrightarrow X^{*}$ is principle.

(ii) $S$ is right $\varphi$-amenable if, for each Banach $S$-bimodule $X \in \mathcal{M}_{\varphi}^{S}$, every bounded derivation $D: S \longrightarrow X^{*}$ is principle.

(iii) $S$ is $\varphi$-amenable if it is both left and right $\varphi$-amenable.

In this paper, we suppose that $0 \neq \varphi \in \Delta_{S}(S)$. The following result is one of the main results of this paper, indeed, this paper results are depend on.

Theorem 2.2. Let $S$ be a topological semigroup and $\varphi \in \Delta_{S}(S)$. Then the following statements are equivalent

(i) $S$ is left (right) $\varphi$-amenable; 
(ii) there is a bounded linear functional $m$ in $\operatorname{RUC}(S)^{*}\left(L U C(S)^{*}\right)$, such that $m(\varphi)=1$ and $m(f \cdot s)=\varphi(s) m(f)(m(s \cdot f)=\varphi(s) m(f))$, for all $s \in S$ and $f \in R U C(S)(f \in L U C(S))$.

Proof. (i) $\Longrightarrow$ (ii) Let $S$ be left $\varphi$-amenable. Continuity of $\varphi$ implies that the Banach algebra $R U C(S)$ is a Banach $S$-bimodule via

$$
(s \cdot f)(t)=\varphi(s) f(t) \quad \text { and } \quad(f \cdot s)(t)=f(s t),
$$

for all $s, t \in S$ and $f \in R U C(S)$. Clearly, every $\varphi$ belongs to $R U C(S)$. It follows that $\varphi \cdot s=\varphi(s) \varphi$, for all $s \in S$. Moreover,

$$
\begin{aligned}
(\varphi \cdot s)(t) & =\varphi(s) \varphi(t)=\varphi(t) \varphi(s)=\varphi(t s) \\
& =\varphi(s) \varphi(t),
\end{aligned}
$$

for all $s, t \in S$. Therefore, for every $s \in S$,

$$
s \cdot \varphi=\varphi \cdot s=\varphi(s) \varphi .
$$

Thus $\mathbb{C} \varphi$ is a closed $S$-subbimodule of $R U C(S)$. Consider the quotient Banach $S$-bimodule $X=R U C(S) / \mathbb{C} \varphi$. Then put $Y=X^{*} \cong\left\{f \in R U C(S)^{*}: f(\varphi)=0\right\} \subseteq$ $R U C(S)^{*}$. Let $\Phi_{0} \in R U C(S)^{*} \backslash Y$ such that $\Phi_{0}(\varphi)=1$ and let $\delta_{\Phi_{0}}: S \longrightarrow Y$ be as follows

$$
\delta_{\Phi_{0}}(s)=s \cdot \Phi_{0}-\Phi_{0} \cdot s=s \cdot \Phi_{0}-\varphi(s) \Phi_{0} .
$$

Clearly, $\delta_{\Phi_{0}}$ is a bounded derivation. On the other hand, $S$ is left $\varphi$-amenable, then there is an element $\mathfrak{x}$ in $Y$ such that

$$
s \cdot \Phi_{0}-\varphi(s) \Phi_{0}=\delta_{\Phi_{0}}(s)=s \cdot \mathfrak{x}-\varphi(s) \mathfrak{x},
$$

for all $s \in S$. Let $m=\Phi_{0}-\mathfrak{x}$. Clearly $m \in R U C(S)^{*}, m(\varphi)=1$ and (2.2) implies $m(f \cdot s)=\varphi(s) m(f)$, for all $s \in S$ and $f \in R U C(S)$. Similarly, we can prove right $\varphi$-amenability.

(ii) $\Longrightarrow$ (i) Let $X \in{ }_{\varphi} \mathcal{M}^{S}$ and $D: S \longrightarrow X^{*}$ be a bounded derivation. For any $x \in X$, we define $\omega_{x}: S \longrightarrow \mathbb{C}$ by $\omega_{x}(s)=(D(s))(x)$. Since $D$ is bounded, $\omega_{x}$ is bounded and continuous. Let $t_{\alpha} \longrightarrow t$ in $S$. Then

$$
\begin{aligned}
\left\|\omega_{x} \cdot t_{\alpha}-\omega_{x} \cdot t\right\|_{\infty}= & \sup _{s \in S}\left|\omega_{x}\left(t_{\alpha} s\right)-\omega_{x}(t s)\right|=\sup _{s \in S}\left|\left(D\left(t_{\alpha} s\right)\right)(x)-(D(t s))(x)\right| \\
\leq & \sup _{s \in S}\left|\varphi(s)\left(D\left(t_{\alpha}\right)-D(t)\right)(x)\right|+\sup _{s \in S}\left|(D(s))\left(x \cdot t_{\alpha}-x \cdot t\right)\right| \\
& \longrightarrow 0 .
\end{aligned}
$$

Therefore, (2.3) follows that $\omega_{x} \in R U C(S)$. Let $m$ be a linear functional in $R U C(S)^{*}$ which satisfies (ii). Define a linear functional $f \in X^{*}$ by $m\left(\omega_{x}\right)=f(x)$, for all $x \in X$. Since $D$ is a derivation, we have

$$
\begin{aligned}
\omega_{x \cdot s}(t) & =(D(t))(x \cdot s)=(s \cdot D(t))(x)=(D(s t))(x)-(\varphi(t) D(s))(x) \\
& =\omega_{x}(s t)-(D(s))(x) \varphi(t)=\left(\omega_{x} \cdot s-(D(s))(x) \varphi\right)(t),
\end{aligned}
$$


for all $s, t \in S$ and $x \in X$. Thus (2.4) implies that $\omega_{x \cdot s}=\omega_{x} \cdot s-(D(s))(x) \varphi$. Then

$$
\begin{aligned}
(\varphi(s) f-s \cdot f)(x) & =\varphi(s) f(x)-f(x \cdot s)=\varphi(s) m\left(\omega_{x}\right)-m\left(\omega_{x}\right) \\
& =\varphi(s) m\left(\omega_{x}\right)-m\left(\omega_{x} \cdot s-(D(s))(x) \varphi\right) \\
& =\varphi(s) m\left(\omega_{x}\right)-m\left(\omega_{x} \cdot s\right)+(D(s))(x) m(\varphi) \\
& =(D(s))(x),
\end{aligned}
$$

for all $s \in S$ and $x \in X$. Hence, $D(s)=s \cdot(-f)-\varphi(s)(-f)$, for all $s \in S$. This means that $D$ is principle and $S$ is left $\varphi$-amenable.

The following example shows that a semigroup $S$ may be $\varphi$-amenable $\left(\varphi \in \Delta_{S}(S)\right)$ but it is not amenable, and thereby, we show that the space of $\varphi$-amenable semigroups is wider than amenable semigroups.

Example 2.3. $\quad$ (i) Let $S$ be a left or a right cancellative semigroup with the identity element e such that, for each $s \neq e$, st $=\varphi(t) s$, where $\varphi \in \Delta_{S}(S)$ and let $\operatorname{dim} \ell^{1}(S) \geq 2$. Let $\left\{\mathcal{V}_{\alpha}\right\}$ be a collection of neighborhood basis for e. We construct a net $\left(v_{\alpha}\right)_{\alpha}$ from $\left\{\mathcal{V}_{\alpha}\right\}$ such that $v_{\alpha} \in \mathcal{V}_{\alpha}$ and $\lim _{\alpha} \varphi\left(v_{\alpha}\right)=1$. By passing into a suitable bounded subnet $\left(\eta_{\alpha}\right)_{\alpha} \subseteq\left(v_{\alpha}\right)_{\alpha}$, we can find an element $\mu \in \beta S$ (Stone - Cech compactification of $S$ ) such that $\mu=\lim _{\alpha} \eta_{\alpha}$. The space $\beta S$ is homeomorphic to the character space of $\ell^{\infty}(S)$. Without loss of generality, we can suppose that $\mu$ belongs to character space of $\ell^{\infty}(S)$ and hence it belongs to $\operatorname{RUC}(S)^{*}$. It is easy to check that $\mu$ satisfies condition (ii) of Theorem 2.2. Thus $S$ is left $\varphi$-amenable, but $S$ is not right amenable because $\ell^{1}(S)$ is not amenable [10, Theorem 2.3].

(ii) Let $S$ and $T$ be semigroups. Suppose that $S$ acts on $T$ on the left; i.e., assume that there is a semigroup homomorphism $\tau$ from $T$ to $\operatorname{End}(S)$, the set of endomorphisms on $S$, such that, for each $t \in T$ there exists $\tau_{t}: S \longrightarrow S$ such that $\tau_{t_{1}}\left(\tau_{t_{2}}(s)\right)=\tau_{t_{1} t_{2}}(s)$, for all $t_{1}, t_{2} \in T$. Then $S \rtimes_{\tau} T$ is called the semidirect product of $S$ and $T$ with respect to $\tau$. If $S \rtimes_{\tau} T$ is the semigroup consisting of elements of the form $(s, t)$, where $s \in S$ and $t \in T$ equipped with multiplication given by

$$
\left(s_{1}, t_{1}\right)\left(s_{2}, t_{2}\right)=\left(s_{1} \tau_{t_{1}}\left(s_{2}\right), t_{1} t_{2}\right),
$$

for all $\left(s_{1}, t_{1}\right),\left(s_{2}, t_{2}\right) \in S \rtimes_{\tau} T$. The amenability of semidirect product of two semigroups is investigated by Klawe [13].

Let $S$ be a left amenable unital semigroup with the identity element e such that consists of at least two elements, $m_{S}$ be a left invariant mean for $S$ and $T$ be a semigroup. Define $\tau$ from $T$ into $\operatorname{End}(S)$ by $\tau_{t}(s)=e$, for all $s \in S$ and $t \in T$. Thus, $\left(s_{1}, t_{1}\right)\left(s_{2}, t_{2}\right)=\left(s_{1}, t_{1} t_{2}\right)$, for all $\left(s_{1}, t_{1}\right),\left(s_{2}, t_{2}\right) \in S \rtimes_{\tau} T$. Let $\varphi \in \Delta_{T}(T)$, then $\widetilde{\varphi}(s, t)=\varphi(t)$ is a character on $S \rtimes_{\tau} T$. Assume that $T$ is $\varphi$-amenable. According to [13, Remark 3.6], $S \rtimes_{\tau} T$ is not left amenable (even $T$ is left amenable). For every $f \in R U C\left(S \rtimes_{\tau} T\right)$, define $f_{t}(s)=f(s, t)$, for all $s \in S$ and $t \in T$. Clearly, $f_{t} \in R U C(S)$ and define $g(t)=m_{S}\left(f_{t}\right)$, for every $t \in T$. Thus, according to definition, we have $g \in R U C(T)$. Since 
$T$ is left $\varphi$-amenable, Theorem 2.2 implies that there exists $m_{\varphi}$ on $R U C(T)$ that satisfies in the stated conditions. Now define $\mathbf{m}(f)=m_{\varphi}(g)$, for every $f \in R U C\left(S \rtimes_{\tau} T\right)$. Then

$$
\begin{aligned}
\mathbf{m}(f \cdot(s, t)) & =m_{\varphi}\left[m_{S}((f \cdot(s, t))(x, y))\right]=m_{\varphi}\left[m_{S}(f(s, t y))\right] \\
& =m_{\varphi}\left[m_{S}\left(f_{t y}(s)\right)\right]=m_{\varphi}[g(t y)]=m_{\varphi}[(g \cdot t)(y)] \\
& =\varphi(t) m_{\varphi}(g)=\varphi(t) \mathbf{m}(f) \\
& =\widetilde{\varphi}(s, t) \mathbf{m}(f),
\end{aligned}
$$

for all $f \in R U C\left(S \rtimes_{\tau} T\right)$ and $(s, t) \in S \rtimes_{\tau} T$. The above obtained equalities imply that $\mathbf{m}(\widetilde{\varphi})=1$. This means that $S \rtimes_{\tau} T$ is left $\widetilde{\varphi}$-amenable.

For a semigroup $S$, by $1_{S}$ we mean the constant function that $1_{S}(s)=1$, for every $s \in S$. Clearly, $1_{S} \in \Delta_{S}(S)$ and we have the following result that gives a relationship between left (right) $1_{S}$-amenability and left (right) amenability of $S$.

Theorem 2.4. Let $S$ be a topological semigroup, then $S$ is right (left) $1_{S}$-amenable if and only if $S$ is left (right) amenable.

Proof. Let $S$ be right $1_{S}$-amenable and let $R U C(S) \in{ }_{1} \mathcal{M}^{S}$. Let $X=$ $R U C(S) / \mathbb{C} 1_{S}$, then $X \in{ }_{1} \mathcal{M}^{S}$ and $X^{*}$ is canonically isometrically isomorphic with the submodule $\left(\mathbb{C} 1_{S}\right)^{\perp}=\left\{f \in R U C(S)^{*}: f\left(1_{S}\right)=0\right\}$. For $f \in R U C(S)^{*} \backslash\left(\mathbb{C} 1_{S}\right)^{\perp}$, define $D: S \longrightarrow R U C(S)^{*}$ by $D(s)=s \cdot f-f \cdot s$. It is easy to check that $D$ is a derivation. Since $R U C(S) \in{ }_{1} \mathcal{M}^{S}, D(s)=s \cdot f-1_{S}(s) f=s \cdot f-f$, for every $s \in S$. Right $1_{S}$-amenability of $S$ implies that there is an element $g \in\left(\mathbb{C} 1_{S}\right)^{\perp}$ such that $D(s)=s \cdot g-g$, for all $s \in S$. Now, define $h=g-f$. Obviously, $h \neq 0$ and $s \cdot h=h$. This means that $h$ is left $S$-invariant on $\operatorname{RUC}(S)$. The Banach algebra $R U C(S)$ is a $C^{*}$-subalgebra of $\ell^{\infty}(S)$, and Gelfand's Theorem implies that there is a compact Hausdorff space $\Omega$ such that $R U C(S)$ is isometrically $*$-isomorphic to $C(\Omega)$ as $C^{*}$-algebras and $S$-bimodules. Hence, we can suppose that $h$ as a $S$-invariant complex Borel regular measure on $\Omega$. Let $|h|$ be the total variation measure of $h$. Now, define $m=|h| /|h|(\Omega)$, which $m$ is a left $S$-invariant mean on $R U C(S)$. In the other words, $S$ is left amenable.

Conversely, suppose that $S$ is left amenable. Let $m$ be a left invariant mean for $S$. Let $\omega_{x}$ and $f \in X^{*}$ be as in the proof of Theorem 2.2. By (2.4) we have

$$
\omega_{x \cdot s}(t)=\left(\omega_{x} \cdot s-(D(s))(x)\right) \varphi_{S}(t)=\left(\omega_{x} \cdot s-(D(s))(x) 1_{S}\right)(t)
$$

for all $s, t \in S$ and $x \in X$. Thus (2.6) implies that $\omega_{x \cdot s}=\omega_{x} \cdot s-(D(s))(x) 1_{S}$. Then by a similar argument in (2.5), we conclude that $D$ is principle.

Definition 2.5. Let $S$ be a topological semigroup and $\Delta_{S}(S)$ be the character space of $S$. We say that

(i) $S$ is left character amenable if, for each $\varphi \in \Delta_{S}(S)$ and Banach S-bimodule $X \in \mathcal{M}_{\varphi}^{S}$, every bounded derivation $D: S \longrightarrow X^{*}$ is principle.

(ii) $S$ is right character amenable if, for each $\varphi \in \Delta_{S}(S)$ and Banach S-bimodule $X \in{ }_{\varphi} \mathcal{M}^{S}$, every bounded derivation $D: S \longrightarrow X^{*}$ is principle. 
(iii) $S$ is character amenable if it is both left and right character amenable.

In the above statements, if $\varphi \in \Phi_{S}$, then we say that $S$ is left (right) semi-character amenable. Now, we consider character amenability of topological semigroups and we obtain the following result by Theorem 2.4.

Corollary 2.6. Let $S$ be a topological semigroup. If $S$ is right (left) character amenable, then $S$ is left (right) amenable.

Corollary 2.7. Let $S$ be a topological semigroup. If $S$ is character amenable then $S$ is amenable.

Corollary 2.8. Let $S$ be a unital and left or right cancellative semigroup. If $S$ is character amenable then $\ell^{1}(S)$ is amenable.

Proof. Corollary 2.7 implies that $S$ is amenable and [10, Theorem 2.3] completes the proof.

Now, this question arises that: when character amenability of a semigroup $S$ and character amenability of $\ell^{1}(S)$ are equivalent? At this time we do not know in general case, but we have the following result for discrete semigroups.

Theorem 2.9. Let $S$ be a discrete semigroup. Then $S$ is $\varphi$-amenable if and only if $\ell^{1}(S)$ is $\widehat{\varphi}$-amenable.

Proof. Let $\varphi \in \Delta_{S}(S)$ and $S$ be right $\varphi$-amenable. Then $\widehat{\varphi}: \ell^{1}(S) \longrightarrow \mathbb{C}$ defined by $\widehat{\varphi}(f)=\sum_{s \in S} \varphi(s) f(s)$ is a character on $\ell^{1}(S)$. Let $X \in \hat{\varphi} \mathcal{M}^{\ell^{1}(S)}$, and let $D$ : $\ell^{1}(S) \longrightarrow X^{*}$ be a bounded derivation. By the following actions we can see $X \in{ }_{\varphi} \mathcal{M}^{S}$ :

$$
s \cdot x=\delta_{s} \cdot x=\widehat{\varphi}\left(\delta_{s}\right) x=\sum_{t \in S} \varphi(t) \delta_{s}(t) x=\varphi(s) x, \text { and } x \cdot s=x \cdot \delta_{s},
$$

for all $s \in S$ and $x \in X$. Consider the mapping $d: S \longrightarrow X^{*}$ by $d(s)=D\left(\delta_{s}\right)$. Clearly $d$ is a bounded derivation, and since $S$ is right $\varphi$-amenable, there exists $\mathfrak{x} \in X^{*}$ such that $d(s)=s \cdot \mathfrak{x}-\varphi(s) \mathfrak{x}$. This implies that $D(f)=f \cdot \mathfrak{x}-\widehat{\varphi}(f) \mathfrak{x}$, for all $f \in \ell^{1}(S)$. Hence $\ell^{1}(S)$ is right $\widehat{\varphi}$-amenable. We obtain $\ell^{1}(S)$ is left $\widehat{\varphi}$-amenable in a similar way..

Conversely, let $\ell^{1}(S)$ be right $\widehat{\varphi}$-amenable. Suppose that $X \in{ }_{\varphi} \mathcal{M}^{S}$, and $d: S \longrightarrow$ $X^{*}$ is a bounded derivation. We can consider $X$ as a Banach $\ell^{1}(S)$-bimodule via

$$
f \cdot x=\sum_{s \in S} f(s)(s \cdot x)=\sum_{s \in S} \varphi(s) f(s) x=\widehat{\varphi}(f) x, \text { and } x \cdot f=\sum_{s \in S} f(s)(x \cdot s),
$$

for all $f \in \ell^{1}(S)$ and $x \in X$. The derivation $d$ can be extended to a bounded derivation $D: \ell^{1}(S) \longrightarrow X^{*}$ with $d(s)=D\left(\delta_{S}\right)$ (for more details see [5], pp. 737). Since $\ell^{1}(S)$ is right $\widehat{\varphi}$-amenable, there exists $\mathfrak{x} \in X^{*}$ such that $D(f)=f \cdot \mathfrak{x}-\widehat{\varphi}(f) \mathfrak{x}$, for all $f \in \ell^{1}(S)$. Then $d(s)=s \cdot \mathfrak{x}-\varphi(s) \mathfrak{x}$, for all $s \in S$. The proof for the left case is similar.

In the above Theorem, if $\varphi \in \Phi_{S}$ and we replace semi-character amenability instead of character amenability, we can prove the following result.

Corollary 2.10. Let $S$ be a discrete semigroup. Then $S$ is semi-character amenable if and only if $\ell^{1}(S)$ is character amenable. 
By the following Theorem, we characterize Johnson's Theorem as follows:

Theorem 2.11. Let $G$ be a locally compact topological group. Then the following statements are equivalent

(i) $G$ is amenable;

(ii) $G$ is character amenable;

(iii) $L^{1}(G)$ is amenable;

(iv) $L^{1}(G)$ is character amenable.

Proof. (i) $\Longrightarrow$ (ii) follows from [15, Theorem 3.7]. Corollary 2.7, implies (ii) $\Longrightarrow$ (i). (i) $\Longleftrightarrow$ (iii) is Johnson's Theorem, and by [17, Corollary 2.4], we have (iii) $\Longleftrightarrow($ iv).

Let $S$ be a topological semigroup and $f \in \ell^{1}(S)$ is said to be a finite mean, if $f(s) \geq 0$, for every $s \in S,\{s: f(s)>0\}$ is finite and $\|f\|=\sum_{s \in S} f(s)=1$. Day proved that a semigroup $S$ is left amenable if and only if there is a net $\left(f_{\gamma}\right)_{\gamma}$ of finite means such that $\left\|s \cdot f_{\gamma}-f_{\gamma}\right\|_{1} \longrightarrow 0[\mathbf{8}, \mathbf{1 8}$. By a similar argument, we have the following result for $\varphi$-amenability of $S$, where $\varphi \in \Delta_{S}(S)$.

Theorem 2.12. Let $S$ be a topological semigroup and $\varphi \in \Delta_{S}(S)$. Then $S$ is left $\varphi$-amenable if and only if there is a bounded net $\left(f_{\alpha}\right)_{\alpha \in I} \subseteq \ell^{1}(S)$ such that $\| s \cdot f_{\alpha}-$ $\varphi(s) f_{\alpha} \|_{1} \longrightarrow 0$ and its $w^{*}$-limit on $\varphi$ is 1 .

Proof. Assume that $S$ is left $\varphi$-amenable and $m$ is a bounded linear functional that is obtained in the Theorem [2.2. Thus, there is a net $\left(f_{\alpha}\right)_{\alpha \in I} \subseteq \ell^{1}(S)$ such that $w^{*}$-converges to $m$ and $\left\|f_{\alpha}\right\|_{1} \leq\|m\|_{\infty}$. Then

$$
f\left(s \cdot f_{\alpha}-\varphi(s) f_{\alpha}\right)=f_{\alpha}(f \cdot s)-\varphi(s) f_{\alpha}(f) \longrightarrow m(f \cdot s)-\varphi(s) m(f)=0,
$$

for all $f \in R U C(S)$ and $s \in S$. Consider the product space $\ell^{1}(S)^{S}$ that is a locally convex linear topological space with the product of the norm topologies. Now define a linear map $T: \ell^{1}(S) \longrightarrow \ell^{1}(S)^{S}$ by $T(g)=(s \cdot g-\varphi(s) g)_{s \in S}$. Thus, if $S$ is left $\varphi$ amenable, then 0 is in the weak closure of $T$ on the set of finite means such as $f \in \ell^{1}(S)$. Since the set of finite means is convex in $\ell^{1}(S)$ and $\ell^{1}(S)^{S}$ is locally convex, $T$ on this set is convex. This implies that the weak closure of $T$ on the set of finite means equals the closure of it in the given topology on $\ell^{1}(S)^{S}$, that is, the product of the norm topologies. Thus, there is a net $\left(f_{\alpha}\right)_{\alpha \in I} \subseteq \ell^{1}(S)$ such that $\left\|s \cdot f_{\alpha}-\varphi(s) f_{\alpha}\right\|_{1} \longrightarrow 0$ and $f_{\alpha} \stackrel{w^{*}}{\longrightarrow} m$.

Conversely, let there is a net $\left(f_{\alpha}\right)_{\alpha \in I} \subseteq \ell^{1}(S)$ such that $w^{*}$-converges to an element of $R U C(S)^{*}$ namely $m$ such that $m(\varphi)=1$ and $\left\|s \cdot f_{\alpha}-\varphi(s) f_{\alpha}\right\|_{1} \longrightarrow 0$, for every $s \in S$. This means that $s \cdot f_{\alpha}-\varphi(s) f_{\alpha} \longrightarrow 0$ in the weak topology. Thus, similar to (2.7), we have $m(f \cdot s)=\varphi(s) m(f)$, for all $f \in R U C(S)$ and $s \in S$. This shows that $S$ is $\varphi$-amenable.

\section{Some Hereditary Properties}

This section deals with the stability properties of $\varphi$-amenability of topological semigroups and groups. We prove these properties via Theorem 2.2 , 
Proposition 3.1. Let $S, T$ be semigroups, $\theta: S \longrightarrow T$ be a continuous and onto semigroups homomorphism. Let $\psi \in \Delta_{T}(T)$, and let $S$ be left (right) $(\psi \circ \theta)$-amenable. Then $T$ is left (right) $\psi$-amenable.

Proof. Let $X \in{ }_{\psi} \mathcal{M}^{T}$ and $D: T \longrightarrow X^{*}$ be a bounded derivation. Then we can see $X$ as an element of $\psi_{\%} \mathcal{M}^{S}$ by the following actions

$$
s \cdot x=\theta(s) \cdot x=\psi(\theta(s)) x, \text { and } x \cdot s=x \cdot \theta(s),
$$

for all $s \in S$ and $x \in X$. Define $D \circ \theta: S \longrightarrow X^{*}$. Obviously, $D \circ \theta$ is a bounded derivation. Thus, there exists $\mathfrak{x} \in X^{*}$ such that

$$
(D \circ \theta)(s)=s \cdot \mathfrak{x}-\psi(\theta(s)) \mathfrak{x},
$$

for all $s \in S$. Since $\theta$ is onto, we have

$$
D(t)=t \cdot \mathfrak{x}-\psi(t) \mathfrak{x}
$$

for all $t \in T$. Similarly, we can prove if $S$ is left $\psi \circ \theta$-amenable, then $T$ is left $\psi$ amenable.

Corollary 3.2. Let $S$ be a topological semigroup, $L$ be a closed ideal in $S$ and $\varphi \in \Delta_{S}(S)$ such that $\left.\varphi\right|_{L} \neq 0$. If $S$ is $\varphi$-amenable, then $L$ is $\left.\varphi\right|_{L}$-amenable.

Corollary 3.3. Let $G$ be a locally compact group, $H$ be a closed normal subgroup of $G$ and $\varphi \in \Delta_{G / H}(G / H)$. If $G$ is $(\varphi \circ \theta)$-amenable, where $\theta: G \longrightarrow G / H$ is the canonical homomorphism, then $G / H$ is $\varphi$-amenable.

It is well-known that the quotient group of an amenable group $G$ by a closed normal subgroup $H$ is amenable and moreover $H$ is amenable as a group. By these facts, Theorem 2.11 and Proposition 3.1, we have the following result:

Corollary 3.4. Let $G$ be a locally compact group and $H$ be a closed normal subgroup of $G$. Then $G$ is character amenable if and only if $H$ and $G / H$ are character amenable.

Let $S$ and $T$ be semigroups. Then $S \times T$ is a semigroup with the operation

$$
\left(s_{1}, t_{1}\right)\left(s_{2}, t_{2}\right)=\left(s_{1} s_{2}, t_{1} t_{2}\right)
$$

for all $s_{1}, s_{2} \in S$ and $t_{1}, t_{2} \in T$. Define $\pi_{S}: S \times T \longrightarrow S$ and $\pi_{T}: S \times T \longrightarrow T$ by $\pi_{S}(s, t)=s$ and $\pi_{T}(s, t)=t$, respectively, for all $s \in S$ and $t \in T$. Clearly, both $\pi_{S}$ and $\pi_{T}$ are continuous and onto semigroups homomorphisms.

Theorem 3.5. Let $S$ and $T$ be two topological semigroups. If $S \times T$ is left (right) character amenable, then $S$ and $T$ are left (right) character amenable.

Proof. Let $S \times T$ be left (right) character amenable. Let $\varphi \in \Delta_{S}(S)$ be an arbitrary and $\pi_{S}$ be as above. Then $\varphi \circ \pi_{S} \in \Delta_{S \times T}(S \times T)$ and Proposition 3.1 implies that $S$ is left (right) $\varphi$-amenable. This shows that $S$ is character amenable, because $\varphi$ was arbitrary. Similarly one can see that $T$ is character amenable.

We consider the converse of the above Theorem in the special case as follows: 
Theorem 3.6. Let $S, T$ be two topological semigroups, $\varphi \in \Delta_{S}(s)$ and $\psi \in \Delta_{T}(T)$. If $S$ is left (right) $\varphi$-amenable and $T$ is left (right) $\psi$-amenable, then $S \times T$ is left (right) $(\varphi, \psi)$-amenable.

Proof. Suppose that $S$ is left $\varphi$-amenable, $T$ is left $\psi$-amenable, $m_{S}$ and $m_{T}$ are the bounded functionals obtained from Theorem 2.2. For each $f \in R U C(S \times T)$ and $(s, t) \in S \times T$, we can define $f_{s} \in R U C(T)$ and $g \in R U C(S)$ as follows

$$
f_{s}(t)=f(s, t) \quad \text { and } \quad g(s)=m_{T}\left(f_{s}(t)\right) .
$$

Now, define $m$ on $R U C(S \times T)$ by $m(f)=m_{S}(g)$, for all $f \in R U C(S \times T)$. Then

$$
\begin{aligned}
m(f \cdot(s, t)) & =m_{S}\left[m_{T}((f \cdot(s, t))(x, y))\right]=m_{S}\left[m_{T}(f(s x, t y))\right] \\
& =m_{S}\left[m_{T}\left(f_{s x}(t y)\right)\right]=m_{S}\left[m_{T}\left(\left(f_{s x} \cdot t\right)(y)\right)\right] \\
& =m_{S}\left[\psi(t) m_{T}\left(f_{s x}(y)\right)\right]=\psi(t) m_{S}[g(s x)] \\
& =\psi(t) m_{S}[(g \cdot s)(x)]=\varphi(s) \psi(t) m_{S}[g(x)] \\
& =\varphi(s) \psi(t) m_{S}\left[m_{T}(f(x, y))\right] \\
& =\varphi(s) \psi(t) m(f)
\end{aligned}
$$

for all $f \in R U C(S \times T)$ and $(s, t) \in S \times T$. Clearly, $(\varphi, \psi) \in R U C(S \times T)$ and the above obtained result follows that $m((\varphi, \psi))=1$. Thus, Theorem 2.2 implies that $S \times T$ is left $(\varphi, \psi)$-amenable.

An involution on a topological semigroup $S$ is a map $*$ from $S$ into $S$ such that, the images of $s, t \in S$ are denoted by $s^{*}$ and $t^{*}$, respectively, $s=\left(s^{*}\right)^{*},(s t)^{*}=t^{*} s^{*}$ and $*$ is a continuous map; see [2, 3] for more results related to topological semigroups with involution and characters on them. Let $f \in L U C(S)$ or $R U C(S)$, we set $\widetilde{f}(s)=f\left(s^{*}\right)$, for all $s \in S$.

Theorem 3.7. Let $S$ be a discrete semigroups with involution $*$ and $\varphi \in \Delta_{S}(S)$. If $S$ is left (right) $\widetilde{\varphi}$-amenable, then $S$ is right (left) $\varphi$-amenable.

Proof. Suppose that $S$ is left (right) $\widetilde{\varphi}$-amenable. Then Theorem 2.2 implies that there is a bounded linear functional $m$ in $\ell^{\infty}(S)^{*}$ such $m(\widetilde{\varphi})=1$ and $m\left(f \cdot s^{*}\right)=$ $\widetilde{\varphi}\left(s^{*}\right) m(f)$, for all $f \in \ell^{\infty}(S)$. Let $f \in \ell^{\infty}(S)$ and define $m^{\prime}(f)=m(\widetilde{f})$. Since the mapping $f \longmapsto \widetilde{f}$ is linear, $m^{\prime}$ is linear and bounded. Furthermore, $m^{\prime}(\varphi)=m(\widetilde{\varphi})=1$ and $m^{\prime}(f) \geq 0$, for all $f \in \ell^{\infty}(S)$.

Moreover, for all $f \in \ell^{\infty}(S)$ and $s, t \in S$, we have

$$
\begin{aligned}
(s \cdot \tilde{f})(t) & =(s \cdot f)\left(t^{*}\right)=f\left(t^{*} s\right)=f\left(\left(s^{*} t\right)^{*}\right) \\
& =\widetilde{f}\left(s^{*} t\right)=\left(\tilde{f} \cdot s^{*}\right)(t) .
\end{aligned}
$$

This shows that $s \cdot \tilde{f}=\tilde{f} \cdot s^{*}$ for all $f \in \ell^{\infty}(S)$ and $s \in S$. Then

$$
\begin{aligned}
m^{\prime}(s \cdot f) & =m(s \cdot \tilde{f})=m\left(\tilde{f} \cdot s^{*}\right)=\widetilde{\varphi}\left(s^{*}\right) m(\widetilde{f}) \\
& =\varphi(s) m^{\prime}(f),
\end{aligned}
$$

for all $f \in \ell^{\infty}(S)$ and $s \in S$. Thus, $S$ is right $\varphi$-amenable. 
Assume that $S$ is a semigroup and $\varphi \in \Delta_{S}(S)$. Let $T$ be a subsemigroup of $S$ and $S$ is left (right) $\varphi$-amenable. We denote $\varphi$ by $\left.\varphi\right|_{T}$ on $T$, clearly, it is a character on $T$, but, maybe $T$ is not left (right) $\varphi_{T}$-amenable. In other words, there is a subsemigroup of $\varphi$-amenable semigroup is not $\varphi$-amenable. Moreover, there is a subsemigroup $T$ of semigroup $S$ and $\varphi \in \Delta_{T}(T)$ such that $T$ is $\varphi$-amenable and $\widetilde{\varphi}$-amenability of $S$ does not sense, where $\widetilde{\varphi}$ is the extension of $\varphi$ on $S$. The following example shows the above statements are true.

Example 3.8. $\quad$ (i) Let $S$ be a semigroup without zero element o. Following [6], we denote the semigroup formed by adjoining o to $S$ by $S^{o}$ and $S$ becomes a subsemigroup of $S^{o}$. Then the only character on $S^{o}$ is $1_{S^{o}} \in \Delta_{S^{o}}\left(S^{o}\right)$. Let $S$ be not left $1_{S}$-amenable. Define $m(f)=f(o)$, for all $f \in R U C\left(S^{o}\right)$. Thus, $S^{o}$ is left $1_{S^{o}-a m e n a b l e .}$

(ii) Let $S$ be a semigroup without zero element o and $\varphi \in \Delta_{S}(S)$ such that $1_{S} \neq \varphi$. If $S$ is $\varphi$-amenable, then according to (i) and by this fact that $\varphi$ has not any extension such as $\widetilde{\varphi}$ on $S^{o}, S^{o}$ is not $\widetilde{\varphi}$-amenable.

Definition 3.9. Let $S$ be a topological semigroup, $T$ be a right thick susbemigroup of $S$ and $\varphi \in \Delta_{S}(S)$. We say that $T$ is strongly left $\left.\varphi\right|_{T}$-amenable if there is a bounded linear functional $m$ on $L U C(T)$ such that (i) $m\left(\left.\varphi\right|_{T}\right)=1$ and (ii) $m(s \cdot f)=\varphi(s) m(f)$, for all $f \in L U C(T), s \in S$. Similarly, one can define the strongly right $\left.\varphi\right|_{T}$-amenability for the left thick susbemigroup $T$ of $S$.

Theorem 3.10. Let $S$ be a topological semigroup, $T$ be a left (right) thick susbemigroup of $S$ and $\varphi \in \Delta_{S}(S)$. Then $T$ is strongly right (left) $\varphi_{T}$-amenable if and only if $S$ is right (left) $\varphi$-amenable.

Proof. We prove the right case and the left case is similar. Assume that $T$ is strongly right $\left.\varphi\right|_{T}$-amenable. Define $\Phi: L U C(S) \longrightarrow L U C(T)$ by $\Phi(f)=\left.f\right|_{T}$. Clearly, $\Phi$ is a bounded linear map and consider $\Phi^{*}: L U C(T)^{*} \longrightarrow L U C(S)^{*}$. By Theorem 2.2. there is a bounded linear functional $m$ in $L U C(T)^{*}$ such that $m\left(\left.\varphi\right|_{T}\right)=1$ and $m(t \cdot f)=\left.\varphi\right|_{T}(t) m(f)$, for all $f \in L U C(T)$ and $t \in T$. We claim that $\mathbf{m}=\Phi^{*}(m)$ is a bounded linear functional for $S$ that satisfies the condition (ii) of Theorem 2.2. Since $T$ is left thick, for all $f \in L U C(S), s \in S$ and $t \in T$, we have

$$
\Phi(s \cdot f)(t)=\left.(s \cdot f)\right|_{T}(t)=\left.f\right|_{T}(t s)=(s \cdot \Phi(f))(t) .
$$

This implies that $\Phi(s \cdot f)=s \cdot \Phi(f)$, for all $f \in L U C(S)$ and $s \in S$. Then

$$
\begin{aligned}
\mathbf{m}(s \cdot f) & =\Phi^{*}(m)(s \cdot f)=m(\Phi(s \cdot f))=m(s \cdot \Phi(f))=\varphi(s) m(\Phi(f)) \\
& =\varphi(s) \mathbf{m}(f),
\end{aligned}
$$

for all $f \in L U C(S)$ and $s \in S$. Moreover,

$$
\mathbf{m}(\varphi)=\Phi^{*}(m)(\varphi)=m(\Phi(\varphi))=m\left(\varphi_{T}\right)=1 .
$$

The relations (3.1) and (3.2) follow that $S$ is right $\varphi$-amenable.

Consider the canonical bounded linear map $\Psi: L U C(T) \longrightarrow L U C(S)$ such that $\left.\Psi(f)\right|_{S \backslash T}=0$, for all $\varphi_{T} \neq f \in L U C(T)$ and $\Psi\left(\varphi_{T}\right)=\varphi$. Let $\mathbf{m}$ be a linear functional 
defined on $\operatorname{LUC}(S)$ that satisfies Theorem 2.2. We now show that $m=\Psi^{*}(\mathbf{m})$ is a $\varphi$-mean for $T$.

For an arbitrary $f \in L U C(T), s \in S$ and $t \in T$,

$$
(\Psi(s \cdot f)-s \cdot \Psi(f))(t)=(s \cdot f)(t)-\Psi(f)(t s)=f(t s)-f(t s)=0 .
$$

Hence, $\left.(\Psi(s \cdot f)-s \cdot \Psi(f))\right|_{T}=0$, and

$$
|(s \cdot f)(t)-s \cdot \Psi(f)(t)| \leq\|\Psi(s \cdot f)-s \cdot \Psi(f)\|_{\infty} \chi_{S \backslash T},
$$

where $\chi_{S \backslash T}$ is the characteristic function on $S \backslash T$. Thus,

$$
\mathbf{m}(\Psi(s \cdot f))=\mathbf{m}(s \cdot \Psi(f)) \quad(f \in L U C(T), s \in S) .
$$

Therefore,

$$
\begin{aligned}
m(s \cdot f) & =\Psi^{*}(\mathbf{m})(s \cdot f)=\mathbf{m}(\Psi(s \cdot f))=\mathbf{m}(s \cdot \Psi(f))=\varphi(s) \mathbf{m}(\Psi(f)) \\
& =\varphi(s) m(f)
\end{aligned}
$$

and

$$
m\left(\varphi_{T}\right)=\Psi^{*}(\mathbf{m})\left(\varphi_{T}\right)=\mathbf{m}\left(\Psi\left(\varphi_{T}\right)\right)=\mathbf{m}(\varphi)=1,
$$

for all $f \in L U C(T)$ and $s \in S$. Thus, $T$ is strongly right $\varphi_{T}$-amenable.

We finish this section with the following result:

Proposition 3.11. Let $\left\{S_{\alpha}\right\}_{\alpha \in I}$ be a family of closed subsemigroups of topological semigroup $S$ such that $S_{\alpha}$ is left (right) $\varphi_{\alpha}$-amenable, for each $\alpha \in I$, where $\varphi_{\alpha} \in$ $\Delta_{S_{\alpha}}\left(S_{\alpha}\right)$. Let the following conditions hold:

(i) for every $S_{\alpha}, S_{\beta}$ that are left (right) $\varphi_{\alpha}$-amenable and $\varphi_{\beta}$-amenable, respectively, there is a $S_{\gamma}$ such that $S_{\alpha} \cup S_{\beta} \subseteq S_{\gamma}$ and $S_{\gamma}$ is $\varphi_{\gamma}$-amenable.

(ii) $S=\overline{\bigcup_{\alpha \in I} S_{\alpha}}$.

Let $\varphi$ be a function on $S$ such that

$$
\varphi(s t)= \begin{cases}\varphi_{\alpha}(s t) & \text { if } s, t \in S_{\alpha} \\ \varphi_{\gamma}(s t) & \text { if } s \in S_{\alpha}, t \in S_{\beta} \text { and } S_{\alpha} \cup S_{\beta} \subseteq S_{\gamma}\end{cases}
$$

Then $\varphi$ is a character on $S$ and $S$ is left (right) $\varphi$-amenable.

Proof. Clearly, $\varphi$ is a character on $S$. Let $m_{\alpha}$ be a bounded linear functional on $R U C\left(S_{\alpha}\right)$, for every $\alpha \in I$, that satisfies Theorem 2.2. Define

$$
m_{\alpha}^{\prime}(f)=m_{\alpha}\left(\left.f\right|_{S_{\alpha}}\right)
$$

for every $f \in R U C(S)$. Let $M_{\alpha}$ be the $w^{*}$-closed set of all bounded linear functionals on $R U C(S)$ such as $m$ such that $m\left(\varphi_{\alpha}\right)=1$ and $m(f \cdot s)=\varphi_{\alpha}(s) m(f)$, for all $f \in R U C(S)$ and $s \in S_{\alpha}$. According to the definition of $m_{\alpha}^{\prime}$, it belongs to $M_{\alpha}$. This means that $M_{\alpha}$ is not empty and it is obvious that the augmentation character $1_{S}$ is in $R U C(S)$. These imply that $\bigcap_{\alpha \in I} M_{\alpha}$ is not empty. Now, let $\mathbf{m} \in \bigcap_{\alpha \in I} M_{\alpha}$. Then the case (i) and definition of $\varphi$ together imply that $\mathbf{m}(\varphi)=1$. Moreover, the cases (i), (ii) and definition of $\varphi$ together imply that for all $f \in R U C(S)$ and $s \in \bigcup_{\alpha \in I} S_{\alpha}$, there exists $\gamma \in I$ such that $s \in S_{\gamma}$ and

$$
\mathbf{m}(f \cdot s)=\varphi_{\gamma}(s) m_{\gamma}\left(\left.f\right|_{S_{\gamma}}\right)=\varphi(s) \mathbf{m}(f) .
$$


Since $f \in R U C(S)$, for every $s \in S$ and $\varepsilon>0$, there is a net $\left(t_{\beta}\right)_{\beta} \subseteq \bigcup_{\alpha \in I} S_{\alpha}$ such that $\left\|(f \cdot s)-\left(f \cdot t_{\beta}\right)\right\|_{\infty}<\varepsilon / 2\|\mathbf{m}\|$ and $\left|\varphi\left(t_{\beta}\right)-\varphi(s)\right|<\varepsilon / 2\|\mathbf{m}\|$. Then (3.6) implies that

$$
\begin{aligned}
|\mathbf{m}(f \cdot s)-\varphi(s) \mathbf{m}(f)| & =\left|\mathbf{m}(f \cdot s)-\mathbf{m}\left(f \cdot t_{\beta}\right)+\mathbf{m}\left(f \cdot t_{\beta}\right)-\varphi(s) \mathbf{m}(f)\right| \\
& =\left|\mathbf{m}(f \cdot s)-\mathbf{m}\left(f \cdot t_{\beta}\right)+\varphi\left(t_{\beta}\right) \mathbf{m}(f)-\varphi(s) \mathbf{m}(f)\right| \\
& \leq\left|\mathbf{m}(f \cdot s)-\mathbf{m}\left(f \cdot t_{\beta}\right)\right|+\left|\varphi\left(t_{\beta}\right)-\varphi(s) \| \mathbf{m}(f)\right| \\
& \leq\|\mathbf{m}\|\left\|(f \cdot s)-\left(f \cdot t_{\beta}\right)\right\|_{\infty}+\left|\varphi\left(t_{\beta}\right)-\varphi(s)\right|\|\mathbf{m}\| \\
& <\varepsilon .
\end{aligned}
$$

Since $\varepsilon$ was arbitrary, $\mathbf{m}(f \cdot s)=\varphi(s) \mathbf{m}(f)$, for all $f \in R U C(S)$ and $s \in S$. This means that $S$ is left $\varphi$-amenable.

\section{4. $\varphi$-Ergodic Properties}

Let $S$ be a topological semigroup, $X$ be a Banach space and $\mathbf{B}(X)$ be the Banach space of all bounded operators on $X$. An antirepresentation of $S$ on $X$ is a function $F: s \longmapsto F_{s}$ such that $F_{s t}=F_{t} F_{s}$, for each $s, t \in S$. For each $s \in S$, define $L=\ell(s)=\ell_{s}$ in $\mathbf{B}(C(S))$ by $L f(t)=f(s t)$, for all $t \in S$ and $f \in C(S)$. The function $\ell$ is the antirepresentation of $S$ on $X$.

Let $\varphi \in \Delta_{S}(S)$, Similar to [7], we define the following sets that we work on them in this section:

$$
\begin{aligned}
& P(\varphi)=\{s \in S: \varphi(s)=1\}, \\
& M_{0}^{\varphi}(\ell)=\left\{x \in X:\left(\ell_{s}-I\right) x=0, s \in P(\varphi)\right\}, \\
& M_{1}^{\varphi}(\ell)=\text { closed linear hull of }\left\{\left(\ell_{s}-I\right) x: x \in X, s \in P(\varphi)\right\}, \\
& M_{\varphi}(\ell)=M_{0}^{\varphi}\left(\ell_{s}\right)+M_{1}^{\varphi}\left(\ell_{s}\right), \\
& N_{x}^{\varphi}(\ell)=\text { the closure of }\left\{\ell_{s}(x): s \in P(\varphi)\right\}, \text { for every } x \in X .
\end{aligned}
$$

Now, we generalize the ergodicity of the antirepresentation $\ell_{s}$ of $S$ into $X$ as follows:

Definition 4.1. Let $S$ be a topological semigroup, $X$ be a Banach space and $\varphi \in$ $\Delta_{S}(S)$. We say that the antirepresentation $\ell$ from $S$ into $X$ is left $\varphi$-ergodic if there is a bounded net $\left(B_{\delta}\right)_{\delta \in I}$ in $\mathbf{B}(X)$ such that

$\left(E_{1}\right) \lim _{\delta} B_{\delta}\left(\ell_{s}-I\right)=0$ in strong operator topology of $\mathbf{B}(X)$, for every $s \in P(\varphi)$.

$\left(E_{2}\right) B_{\delta}(x) \in N_{x}^{\varphi}\left(\ell_{s}\right)$, for each $x \in X$ and $\delta \in I$.

Similarly, we call the antirepresentation $\ell$ from $S$ into $X$ is right $\varphi$-ergodic if there is a bounded net $\left(B_{\delta}\right)_{\delta \in I}$ in $\mathbf{B}(X)$ such that satisfies $\left(E_{2}\right)$ and the following condition:

$\left(E_{3}\right) \lim _{\delta}\left(\ell_{s}-I\right) B_{\delta}=0$ in strong operator topology of $\mathbf{B}(X)$, for every $s \in P(\varphi)$.

If the antirepresentation $\ell$ from $S$ into $X$ is right and left $\varphi$-ergodic, we call it $\varphi$-ergodic.

The following result is the generalization of the obtained results by Eberlein in 4 where the proof is similar and we give it proof for clearness:

Theorem 4.2. Let $S$ be a topological semigroup, $X$ be a Banach space and $\varphi \in$ $\Delta_{S}(S)$. Assume that the antirepresentation $\ell$ from $S$ into $X$ is left $\varphi$-ergodic with $\left(B_{\delta}\right)_{\delta \in I}$ in $\mathbf{B}(X)$ that satisfies the cases $\left(E_{1}\right)$ and $\left(E_{2}\right)$. Then 
(i) $B_{\delta}(x)=x$, for all $x \in M_{0}^{\varphi}(\ell)$ and $\delta \in I$.

(ii) $B_{\delta}(x) \longrightarrow 0$, for every $x \in M_{1}^{\varphi}(\ell)$.

(iii) $\left(B_{\delta}(x)\right)_{\delta \in I}$ is norm convergent to an element of $M_{0}^{\varphi}(\ell) \cap N_{x}^{\varphi}(\ell)$.

(iv) $M_{\varphi}(\ell)=M_{0}^{\varphi}(\ell) \oplus M_{1}^{\varphi}(\ell)$.

(v) $\ell_{s}\left(M_{\varphi}(\ell)\right) \subseteq M_{\varphi}(\ell)$ and $N_{x}^{\varphi}(\ell) \subseteq M_{\varphi}(\ell)$, for all $s \in P(\varphi)$ and $x \in M_{\varphi}(\ell)$.

(vi) $B_{\delta}\left(M_{\varphi}(\ell)\right) \subseteq M_{\varphi}(\ell)$ for all $\delta \in I$.

(vii) suppose that $\pi: M_{\varphi}(\ell) \longrightarrow M_{0}^{\varphi}(\ell)$ is a projection associated with the direct sum decomposition (iv), then $B_{\delta}(x) \longrightarrow \pi(x)$ and $M_{0}^{\varphi}(\ell) \cap N_{x}^{\varphi}(\ell)=\{\pi x\}$, for all $x \in M_{\varphi}(\ell)$.

Proof. (i) If $x \in M_{0}^{\varphi}(\ell)$, then $\ell_{s} x=I x=x$. This implies that $N_{x}^{\varphi}(\ell)=\{x\}$ and consequently, $E_{2}$ leads $B_{\delta}(x)=x$, for every $\delta \in I$.

(ii) Assume that $x \in M_{1}^{\varphi}(\ell)$. Then the case $\left(E_{1}\right)$ together with $\left(B_{\delta}\right)_{\delta \in I}$ is bounded, we have $B_{\delta}(x) \longrightarrow 0$.

(iii) The cases (i) and (ii) together imply this case.

(iv) The cases (i) and (ii) together imply $M_{0}^{\varphi}(\ell) \cap M_{1}^{\varphi}(\ell)=\{0\}$ and this means that $M_{\varphi}(\ell)=M_{0}^{\varphi}(\ell) \oplus M_{1}^{\varphi}(\ell)$.

(v) For all $s, t \in P(\varphi)$ and $x \in X$, we have

$$
\ell_{s}\left(\ell_{t}-I\right)(x)=\left(\ell_{t s}-\ell_{s}\right)(x)=\left(\ell_{t s}-I\right)(x)-\left(\ell_{s}-I\right)(x) \in M_{1}^{\varphi}(\ell),
$$

because $t s \in P(\varphi)$. This means that $\ell_{s}\left(M_{1}^{\varphi}(\ell)\right) \subseteq M_{1}^{\varphi}(\ell)$. Then by applying (i) and (iv), we have $\ell_{s}\left(M_{\varphi}(\ell)\right) \subseteq M_{\varphi}(\ell)$.

For showing that $N_{x}^{\varphi}(\ell) \subseteq M_{\varphi}(\ell)$, for every $x \in M_{\varphi}(\ell)$, pick $x \in M_{\varphi}(\ell)$ and let $y \in N_{x}^{\varphi}(\ell)$. According to the definition of $N_{x}^{\varphi}(\ell)$, there is a net $\left(s_{\alpha}\right)_{\alpha \in J}$ in $P(\varphi)$ such that $\ell_{s_{\alpha}}(x) \longrightarrow y$. Then (iv) implies that there exist $e \in M_{0}^{\varphi}(\ell)$ and $k \in M_{1}^{\varphi}(\ell)$ such that $x=e+k$. Since $\ell_{s}\left(M_{1}^{\varphi}(\ell)\right) \subseteq M_{1}^{\varphi}(\ell)$ and $M_{1}^{\varphi}(\ell)$ is closed, by (i) we have

$$
y-e=\lim _{\alpha} \ell_{s_{\alpha}}(x-e)=\lim _{\alpha} \ell_{s_{\alpha}}(k) \in M_{1}^{\varphi}(\ell) .
$$

Again by (iv), we conclude that $N_{x}^{\varphi}(\ell) \subseteq M_{\varphi}(\ell)$, for every $x \in M_{\varphi}(\ell)$.

(vi) Apply (v) and $\left(E_{1}\right)$.

(vii) The parts (i) and (ii) imply that $B_{\delta}(x) \longrightarrow \pi(x)$, for all $x \in M_{\varphi}(\ell)$. Let $x \in M_{\varphi}(\ell)$ be arbitrary. Then by the parts (i) and (ii) we have

$$
\pi(x) \in M_{0}^{\varphi}(\ell) \cap N_{x}^{\varphi}(\ell) .
$$

Now, assume that $y \in M_{0}^{\varphi}(\ell) \cap N_{x}^{\varphi}(\ell)$. Since $y \in N_{x}^{\varphi}(\ell)$, there is a net $\left(s_{\alpha}\right)_{\alpha \in J}$ in $P(\varphi)$ such that $\ell_{s_{\alpha}}(x) \longrightarrow y$. Then

$$
y-x=\lim _{\alpha}\left(\ell_{s_{\alpha}}-I\right)(x) \in M_{1}^{\varphi}(\ell) .
$$

This shows that $\pi(y-x)=0$. Thus, $\pi(x)=y$ and this completes the proof.

The above Theorem immediately follows the following result that is a generalization of the obtained results in [7].

Proposition 4.3. Let $S$ be a topological semigroup, $X$ be a Banach space and $\varphi \in \Delta_{S}(S)$. Assume that the antirepresentation $\ell$ from $S$ into $X$ is $\varphi$-ergodic with 
$\left(B_{\delta}\right)_{\delta \in I}$ in $\mathbf{B}(X)$ that satisfies $\left(E_{1}\right)$, $\left(E_{2}\right)$ and $\left(E_{3}\right)$. Then the following statements hold.

(i) $M_{\varphi}(\ell)$ is closed in $X$.

(ii) If $N_{x}^{\varphi}(\ell)$ is weakly compact, for every $x \in X$, then $M_{\varphi}(\ell)=X$.

Proof. (i) Theorem 4.2(iii) implies that $\left(B_{\delta}\right)_{\delta \in I}$ is norm convergent to an element of $M_{0}^{\varphi}(\ell) \cap N_{x}^{\varphi}(\ell)$, for every $x \in M_{\varphi}(\ell)$. This follows that $\left(B_{\delta}\right)_{\delta \in I}$ is weakly convergent to an element of $M_{0}^{\varphi}(\ell) \cap N_{x}^{\varphi}(\ell)$, for every $x \in M_{\varphi}(\ell)$.

Let $x \in X$ and $B_{\delta} x \longrightarrow y$ weakly in $X$, for some $y \in X$. We shall show that $y \in M_{\varphi}(\ell)$. For any $s \in P(\varphi)$ and $T \in X^{*}$, we have

$$
\begin{aligned}
T\left(\ell_{s}\right)(y) & =\lim _{\delta} T\left(\ell_{s}\right)\left(B_{\delta}(x)\right)=\lim _{\delta}\left[T\left(\ell_{s}-I\right)\left(B_{\delta}(x)\right)+T\left(B_{\delta}(x)\right)\right] \\
& =T(y) .
\end{aligned}
$$

This means that $\ell_{s}(y)=y$ and consequently, $y \in M_{0}^{\varphi}(\ell)$. Moreover, for every $x \in X, N_{x}^{\varphi}(\ell)$ is convex and norm closed in $X$, so, it is weakly closed and $y \in N_{x}^{\varphi}(\ell)$. Thus, $y \in M_{0}^{\varphi}(\ell) \cap N_{x}^{\varphi}(\ell)$. Then

$$
x=y+(x-y) \in M_{0}^{\varphi}(\ell)+M_{1}^{\varphi}(\ell)=M_{\varphi}(\ell) .
$$

Hence, $M_{\varphi}(\ell)$ is closed in $X$.

(ii) Assume that $N_{x}^{\varphi}(\ell)$ is weakly compact, for every $x \in X$. Let $y \in X$ be an arbitrary element. The fact $\left(B_{\delta}\right)_{\delta \in I} \in N_{x}^{\varphi}(\ell)$ implies that there is a subnet $\left(B_{\delta_{\gamma}}\right)$ of $\left(B_{\delta}\right)_{\delta \in I}$ such that $B_{\delta_{\gamma}}(y)$ is weakly convergent in $N_{x}^{\varphi}(\ell)$. Now, if we replace $\left(B_{\delta}\right)_{\delta \in I}$ by $\left(B_{\delta_{\gamma}}\right)$ in the proof of the part (i), then $y \in M_{\varphi}(\ell)$. Thus, $X=M_{\varphi}(\ell)$.

Let $S$ be a locally compact topological semigroup and $X$ be a right Banach $S$ module. Then we can see $X$ as a right Banach $\ell^{1}(S)$-module as follows:

$$
x f=\int_{S} x s \mathrm{~d} f(s),
$$

for all $x \in X$ and $f \in \ell^{1}(S)$; see [21, Proposition 5.6] for more details. Furthermore, for every $T \in X^{*}$, we define

$$
T(x f)=f(T x),
$$

for all $x \in X$ and $f \in \ell^{1}(S)$. We now give a relation between left $\varphi$ amenability and left $\varphi$-ergodicity of an antirepresentions as follows:

Theorem 4.4. Let $S$ be a locally compact topological semigroup, $X$ be a Banach space, $\varphi \in \Delta_{S}(S)$ and $\ell$ be the right action of $S$ on $X$ i.e. $\ell_{s}(x)=x$ s. If $N_{x}^{\varphi}(\ell)$ is weakly compact, for every $x \in X$, then the following statements are equivalent:

(i) $S$ is left $\varphi$-amenable.

(ii) the antirepresention $\ell$ is left $\varphi$-ergodic.

Proof. (i) $\Longrightarrow$ (ii) Assume that $\ell$ is an antirepresentaion from $S$ into $X$. Theorem 2.12 implies that there is a bounded net $\left(f_{\alpha}\right)_{\alpha \in I} \subseteq \ell^{1}(S)$ such that $\left\|s \cdot f_{\alpha}-\varphi(s) f_{\alpha}\right\|_{1} \longrightarrow$ 
0 and its $w^{*}-\lim$ on $\varphi$ is 1 . Put $B_{\alpha}=\ell_{f_{\alpha}}$, for all $\alpha \in I$. Clearly, $B_{\alpha}$ is bounded in $\mathbf{B}(X)$. Moreover, by noting that for $s \in S$ and $x \in X, x \delta_{s}=x s$, then

$$
\begin{aligned}
\left\|B_{\alpha}\left(\ell_{s}-I\right)\right\| & =\left\|B_{\alpha}\left(\ell_{\delta_{s}}-I\right)\right\|=\left\|\ell_{f_{\alpha}} \ell_{\delta_{s}}-\ell_{f_{\alpha}}\right\|=\left\|\ell_{\delta_{s} * f_{\alpha}}-\ell_{f_{\alpha}}\right\|=\left\|\ell_{s \cdot f_{\alpha}-f_{\alpha}}\right\| \\
& \leq\|\ell\|\left\|s \cdot f_{\alpha}-f_{\alpha}\right\|_{1} .
\end{aligned}
$$

Therefore, (4.2) implies that

$$
\left\|B_{\alpha}\left(\ell_{s}-I\right)\right\| \leq\|\ell\|\left\|s \cdot f_{\alpha}-\varphi(s) f_{\alpha}\right\|_{1} \longrightarrow 0,
$$

for all $s \in P(\varphi)$. Now, we must show that $B_{\alpha}$ satisfies $\left(E_{2}\right)$ i.e., $B_{\alpha} \in N_{x}^{\varphi}(\ell)$, for all $x \in X$ and $\alpha \in I$. Note that

$$
N_{x}^{\varphi}(\ell) \subseteq \overline{\left\{\ell_{f}(x): f \in \ell^{1}(S) \text { such that } f \text { is a finite mean }\right\}}=K .
$$

We claim that $\subseteq$ must be equality in the case that $N_{x}^{\varphi}(\ell)$ is weakly compact, for every $x \in X$. Assume towards a contradiction that there esists $f \in \ell^{1}(S)$ such that $\ell_{f} \notin N_{x}^{\varphi}(\ell)$. Thus, there exist $T \in X^{*}$ and $r \in \mathbb{R}$ such that

$$
\boldsymbol{\operatorname { R e }} T\left(\ell_{s}(x)\right)<r<\boldsymbol{\operatorname { R e }} T\left(\ell_{f}(x)\right)
$$

for every $s \in S$. On the other hand,

$$
\begin{aligned}
\operatorname{Re} T\left(\ell_{f}(x)\right) & =\operatorname{Re} T\left(\int_{S} x s \mathrm{~d} f(s)\right)=\int_{S} \boldsymbol{\operatorname { R e }} T\left(\ell_{s}(x)\right) \mathrm{d} f(s) \\
& <\boldsymbol{\operatorname { R e }} T\left(\ell_{s}(x)\right) .
\end{aligned}
$$

A contradiction. Thus, $N_{x}^{\varphi}(\ell)=K$. This means that $B_{\alpha}$ satisfies $\left(E_{2}\right)$.

(ii) $\Longrightarrow$ (i) Let $X=R U C(S), \varphi \in \Delta_{S}(S)$, and let $\ell$ be an antirepresntation from $S$ into $L U C(S)$ such that

$$
\ell_{s}(f)=f \cdot s=\varphi(s) f
$$

for every $s \in S$ and

$$
\ell_{s}(f)=f \cdot s=\varphi(s) f=f
$$

for every $s \in P(\varphi)$. Then,

$$
\left(\ell_{s}-I\right)(\varphi)=\ell_{s}(\varphi)-\varphi=\varphi \cdot s-\varphi=\varphi(s) \varphi-\varphi=0
$$

for every $s \in P(\varphi)$. This means that $\varphi \in M_{0}^{\varphi}(\ell) \subseteq R U C(S)$ and Theorem 4.2(iv) follows that $\varphi \notin M_{1}^{\varphi}(\ell) \subseteq R U C(S)$. Thus, the Hahn-Banach Theorem implies that there exists $m \in R U C(S)^{*}$ such that $m(\varphi)=1$ and $\left.m\right|_{M_{1}^{\varphi}(\ell)}=0$. Moreover, we have

$$
m(f \cdot s)=\varphi(s) m(f),
$$

for all $s \in S$ and $f \in L U C(S)$. Thus $S$ is left $\varphi$-amenable. 


\section{5. $\varphi$-Amenability and Fixed Point Property}

Let $S$ be a topological semigroup, $C_{r}(S)$ be the space of all bounded real-valued functions on $S$ with supremum norm, $X$ be a translation-invariant closed subalgebra of $C_{r}(S)$ that contains the constant functions, $Y$ be a compact Hausdorff space and $C_{r}(Y)$ be the space of all bounded real-valued continuous functions on $Y$, where $C_{r}(Y)$ has the supremum norm. Assume that $s \longmapsto \lambda_{s}$ is a representation of $S$ by continuous self-maps of $Y$. For every $y \in Y$, we define $T_{y}: C_{r}(Y) \longrightarrow C_{r}(S)$ by $T_{y}(h)(s)=h\left(\lambda_{s} y\right)$, for all $s \in S$ and $h \in C_{r}(Y)$. The representation $\lambda$ is called $D$-representation of $S, X$ on $Y$ if $\left\{y \in Y: T_{y}\left(C_{r}(Y)\right) \subseteq X\right\}$ is dense in $Y$. If $s \longmapsto \lambda_{s}$ is a representation of $S$ by continuous affine self-maps of $Y$, then it is called $D$-representation of $S, X$ on $Y$ by continuous affine maps if $\left\{y \in Y: T_{y}(A(Y)) \subseteq X\right\}$ is dense in $Y$.

The existence of a common fixed point of the family $\lambda_{S}$, whenever $X$ has a left invariant mean is considered by Argabright in [1] and Mitchell in [16. Indeed, Day's fixed point Theorem for topological semigroups is explained and investigated by Argabright and Mitchell works. Namioka in [19] showed that $L U C(S)$ is a translationinvariant subspace of $C_{r}(S)$ [19, Lemma 2] and similarly, one can see that $R U C(S)$ is a translation-invariant subspace of $C_{r}(S)$. We recall the following result:

Theorem 5.1. 16. Theorem 1] Let $S$ be a topological semigroup. Then the following assertions equivalent:

(i) $R U C(S)$ has a multiplicative left invariant mean.

(ii) whenever $S$ acts on a compact Hausdorg space $Y$, where the map $S \times Y \longrightarrow Y$ is jointly continuous, then $Y$ contains a common fixed point of $S$.

Let $S$ be a topological semigroup, $\varphi \in \Delta_{S}(S)$ and $P(\varphi)$ be as defined in the previous section. Clearly, $P(\varphi)$ is a topological subsemigroup of $S$ such $\left.\varphi\right|_{P(\varphi)}=1$. Then by replacing $S$ by $P(\varphi)$ in Theorem 5.1, we have the following result that we give its proof because we use some of the obtained results in the proof, for the last result of this paper:

Corollary 5.2. Let $S$ be a topological semigroup and $\varphi \in \Delta_{S}(S)$. Then the following assertions equivalent:

(i) There is a bounded linear functional $m \in R U C(P(\varphi))^{*}$ such that $m(\varphi)=1$ and $m(f \cdot s)=m(f)$, for all $f \in R U C(P(\varphi))$ and $s \in P(\varphi)$.

(ii) $Y$ contains a common fixed point of $P(\varphi)$ for continuous affine actions of $P(\varphi)$ on compact convex sets of a locally convex linear topological space.

Proof. (i) $\Longrightarrow$ (ii) Let $Y$ be compact convex set of locally convex linear topological space $X$. For each $y \in Y$, we define $T_{y}: C_{r}(Y) \longrightarrow C_{r}(P(\varphi))$ and $R_{y}: S \longrightarrow Y$ by $\left(T_{y} h\right)(s)=h(y s)$ and $R_{y}(s)=y s$, for all $h \in C_{r}(Y)$ and $s \in P(\varphi)$. Then

$$
\left(T_{y} h\right)(s)=h(y s)=\left(h R_{y}\right)(s),
$$

for all $h \in C_{r}(Y)$ and $s \in P(\varphi)$. Thus, $T_{y} h \in C(S)$, because $R_{y}(s)$ is continuous. Now, set $T_{y} h=f$, then

$$
r_{s} f(t)=f(t s)=\left(T_{y} h\right)(t s)=h(y t s),
$$


for all $s, t \in P(\varphi)$. We claim that $f \in R U C(P(\varphi))$. Assume towards a contradiction that $f \notin R U C(S)$ i.e., there exist $s \in P(\varphi)$ and a net $\left(s_{\alpha}\right)_{\alpha \in I} \subseteq P(\varphi)$ such that $s_{\alpha} \longrightarrow s$ but $r_{s_{\alpha}} f$ does not convergent uniformly to $r_{s} f$. This means that there is a positive number $\beta$ and a net $\left(t_{\alpha}\right)_{\alpha \in I} \subseteq P(\varphi)$ such that

$$
\left|h\left(y t_{\alpha} s_{\alpha}\right)-h\left(y\left(t_{\alpha} s\right)\right)\right| \geq \beta
$$

for all $\alpha \in I$. Denote $y t_{\alpha}$ by $y_{\alpha}$. Since $Y$ is compact, $\left(y_{\alpha}\right)_{\alpha \in I}$ has a subnet such as $\left(y_{\gamma}\right)_{\gamma \in I}$ such that converges to some $y^{\prime} \in Y$. By continuity of $h$ and joint continuity of action of $P(\varphi)$ on $Y$, we have

$$
0<\beta \leq \lim _{\gamma}\left|h\left(y_{\gamma} s_{\gamma}\right)-h\left(y_{\gamma} s\right)\right|=\left|h\left(y^{\prime} s\right)-h\left(y^{\prime} s\right)\right|=0 .
$$

This is a contradiction and so, $f \in R U C(P(\varphi))$. This leads to that $T_{y} C(Y) \subseteq$ $R U C(P(\varphi))$ and consequently, $T_{y} A(Y) \subseteq R U C(P(\varphi))$. Let $m \in R U C(S)^{*}$ such that $m(\varphi)=1$ and $m(f \cdot s)=\varphi(s) m(f)$, for all $f \in R U C(S)$ and $s \in S$. Now; without loss of generality, consider $T_{y}: A(Y) \longrightarrow R U C(P(\varphi))$. Then the adjoint of $T_{y}$ is $T_{y}^{*}: R U C(P(\varphi))^{*} \longrightarrow A(Y)^{*}$. Thus, there exists $y^{\prime} \in Y$ such that $T_{y}^{*} m(h)=h\left(y^{\prime}\right)$, for every $h \in A(Y)$. Then

For $s \in P(\varphi)$, define $\Gamma_{s}: A(Y) \longrightarrow A(Y)$ by $\Gamma_{s} h(y)=h(s y)$, for every $h \in A(Y)$.

$$
\begin{aligned}
\left(T_{y} \Gamma_{s} h\right)(t) & =h(y s t)=T_{y} h(s t) \\
& =\left(T_{y} h \cdot s\right)(t),
\end{aligned}
$$

for all $h \in A(Y)$ and $t \in P(\varphi)$. Moreover, by (5.1) we have

$$
\begin{aligned}
h\left(s y^{\prime}\right) & =\Gamma_{s} h\left(y^{\prime}\right)=\Gamma_{s}\left(T_{y}^{*} m(h)\right)=m\left(T_{y}\left(\Gamma_{s} h\right)\right)=m\left(T_{y} h \cdot s\right) \\
& =\varphi(s) m\left(T_{y} h\right)=m\left(T_{y} h\right)=T_{y}^{*} m(h) \\
& =h\left(y^{\prime}\right),
\end{aligned}
$$

for all $h \in A(Y)$ and $s \in P(\varphi)$. This shows that $s y^{\prime}=y^{\prime}$.

(ii) $\Longrightarrow\left(\right.$ i) Set $X=R U C(P(\varphi))^{*}$ with $w^{*}$-topology and suppose that $Y$ is the compact convex set of all bounded linear functionals on $\operatorname{RUC}(P(\varphi))$ such that, for each $m \in Y, m(\varphi)=1$. Define the affine action $T: P(\varphi) \times Y \longrightarrow Y$ by $T_{s} \mu=l_{s}^{*} \mu$, for all $s \in P(\varphi)$ and $\mu \in Y$, where $l_{s}^{*}$ is the adjoint of $l_{s}$. It is easy to check that $T$ is a uniformly continuous affine action on $Y$. Thus, (ii) implies that there exists $m \in Y$ that is fixed under the affine action $T$ of $P(\varphi)$ such that $l_{s}^{*} m=s \cdot m=m$. This means that $s \cdot m(f)=m(f \cdot s)=m(f)$. Thus, (ii) implies (i).

Remark 5.3. Note that if a topological semigroup $S$ is $\varphi$-amenable, where $\varphi \in$ $\Delta_{S}(S)$, then the condition (ii) holds by the same reasons in the proof of the above Corollary, but, we do not know the converse part holds or not?

Similar to Corollary 5.2, one can consider the conditions weakly right uniformly continuous functions on $S$ instead of right uniformly continuous functions, we do not consider this case in this paper.

Suppose that $E$ is a separated locally convex space and $X$ is a subset of $E$ containing an $n$-dimensional subspace. Let $S$ be a topological semigroup and $\mathcal{T}=\left\{T_{s}: s \in S\right\}$ 
be a representation of $S$ as continuous linear transformations from $E$ into $E$ such that $T_{s}(L)$ is an $n$-dimensional subspace contained in $X$ whenever $L$ is an $n$-dimensional subspace contained in $X$, and there exists a closed $\mathcal{T}$-invariant subspace $H$ in $E$ of codimension $n$ with the property that $x+H \cap X$ is compact and convex, for each $x \in E$. If $S$ is left amenable, then exists an $n$-dimensional subspace $L_{0}$, contained in $X$ such that $T_{s}\left(L_{0}\right)=L_{0}$, for all $s \in S[\mathbf{9}$. Lau generalized the above result and introduced the $\mathcal{P}(n)$ condition for topological semigroups and proved that the condition $\mathcal{P}(1)$ implies the left amenability of topological semigroups [14. Let $X \subseteq E$, following [14] by $\mathcal{L}_{n}(X)$ we mean all $n$-dimensional subspaces of $E$ contained in $X$. We now write $\mathcal{P}(n)$ condition as follows:

$\mathcal{P}(n)$ : Let $S$ be a topological semigroup and $\mathcal{T}=\left\{T_{s}: s \in P(\varphi)\right\}$ be a representation of $P(\varphi)$ as linear operators from $E$ into $E$ jointly continuous on compact convex subsets of $E$. Let $X$ be a subset of $E$ such that there is a closed $\mathcal{T}$-invariant subspace $H$ in $E$ of codimension $n$ with the property that $x+H \cap X$ is compact and convex, for each $x \in E$. If $\mathcal{L}_{n}(X)$ is non-empty and $\mathcal{T}$-invariant, then there exists $L_{0} \in \mathcal{L}_{n}(X)$ such that $T_{s}\left(L_{0}\right)=L_{0}$, for each $s \in P(\varphi)$.

Similar to proof of [14, Theorem 1] and by Corollary [5.2, we have the following result:

Corollary 5.4. Let $S$ be a topological semigroup and $\varphi \in \Delta_{S}(S)$.

(i) If $S$ is left $\varphi$-amenable, then $S$ satisfies $\mathcal{P}(n)$, for each positive integer $n$.

(ii) If $S$ satisfies $\mathcal{P}(1)$, then $S$ is left $\varphi$-amenable.

Let $S$ be a topological semigroup and $\ell: S \times R U C(S)^{*} \longrightarrow R U C(S)^{*}$ be the left action of $S$ on $R U C(S)^{*}$ defined by $(s, m) \longmapsto \ell_{s} m$, for all $m \in R U C(S)^{*}$ and $s \in S$ such that $\ell_{s} I=I$ and $\ell_{s} \ell_{s^{\prime}}=\ell_{s^{\prime} s}$, for all $s, s^{\prime} \in P(\varphi)$, where $I$ is the identity element of $R U C(S)^{*}$. Thus, $\ell$ on $P(\varphi)$ is an antirepresentation. Suppose that $X \subseteq R U C(S)^{*}$ is a subspace that contains $I$ and all elements of $R U C(S)^{*}$ such as $m$ such that $m(\varphi)=1$. A mean $\mu$ on $X$ is called $P(\varphi)$-invariant under $\ell$ if $\mu \ell_{s}=\mu$, for every $s \in P(\varphi)$ and we say that $\mu$ on $X$ is $S_{\varphi}$-invariant under $\ell$ if $\mu \ell_{s}=\varphi(s) \mu$, for every $s \in S$. Clearly, every $S_{\varphi}$-invariant mean under $\ell$ is $P(\varphi)$-invariant.

Definition 5.5. Let $S$ be a topological semigroup. We say that $S$ has the HahnBanach Theorem Property if, for each continuous left action $\ell: S \times R U C(S)^{*} \longrightarrow$ $R U C(S)^{*}$ and every $P(\varphi)$-invariant subspace $X$ of $R U C(S)^{*}$ that contains $I$ and all elements of $R U C(S)^{*}$ such as $m$ such that $m(\varphi)=1$, every $P(\varphi)$-invariant mean $\mu$ on $X$ can be extended to a $S_{\varphi}$-invariant mean $\widetilde{\mu}$ on $R U C(S)^{*}$.

The Hahn-Banach Property of semigroups is studied by Silverman 23 and for a special semigroups namely adjoint semigroups studied by van Neerven [20]. By the following, we characterize left $\varphi$-amenability of $S$ :

Theorem 5.6. Let $S$ be a topological semigroup and $\varphi \in \Delta_{S}(S)$, the following assertions are equivalent:

(i) $S$ is left $\varphi$-amenable.

(ii) $S$ has the Hahn-Banach Theorem Property. 
(iii) for any Banach $S$-submodule $Y$ of $X$, each linear functional in $\bigcap_{s \in S}\left\{y^{*} \in\right.$ $\left.Y^{*}: s \cdot y^{*}=\varphi(s) y^{*}\right\}$ has an extension to a linear functional in $\bigcap_{s \in S}\left\{x^{*} \in\right.$ $\left.X^{*}: s \cdot x^{*}=\varphi(s) x^{*}\right\}$;

(iv) there is a bounded projection from $X^{*}$ onto $\bigcap_{s \in S}\left\{x^{*} \in X^{*}: s \cdot x^{*}=\varphi(s) x^{*}\right\}$ which commutes with any bounded linear operator from $X^{*}$ into $X^{*}$ commuting with the action of $S$ on $X$.

Proof. (i) $\Longrightarrow\left(\right.$ ii) Consider $R U C(S)^{*}$ with $w^{*}$-topology and suppose that $\mu$ is a $P(\varphi)$-invariant mean on a $P(\varphi)$-invariant subspace $X$ of $R U C(S)^{*}$. Let $Y$ be a subspace of $R U C(S)^{*}$, contains all means on $R U C(S)^{*}$ that they are extensions of $\mu$. The HahnBanach Theorem follows that $Y$ is non-empty and $w^{*}$-compactness and convexity of the set of all means on $R U C(S)^{*}$ implies that it is $w^{*}$-compact and convex.

Define the continuous affine action $T: P(\varphi) \times R U C(S)^{* *} \longrightarrow R U C(S)^{* *}$ by $T_{s} \nu=$ $\ell_{s}^{*} \nu$, for all $\nu \in R U C(S)^{* *}$ and $s \in P(\varphi)$, where $\ell_{s}^{*}$ is the adjoint of $\ell_{s}$. Continuity of $\ell_{s}$ implies that the maps $s \longmapsto T_{s} \nu$ and $\mu \longmapsto T_{s} \nu$ are continuous, for all $\nu \in R U C(S)^{*}$ and $s \in P(\varphi)$.

For all $\widetilde{\mu} \in Y$ and $s \in P(\varphi)$,

$$
\ell_{s}^{*} \widetilde{\mu}(I)=\widetilde{\mu}\left(\ell_{s} I\right)=\mu(I)=1 .
$$

Hence, $\widetilde{\mu}$ is a mean on $\operatorname{RUC}(S)^{*}$. Moreover,

$$
\begin{aligned}
\ell_{s}^{*} \widetilde{\mu}(m) & =\widetilde{\mu} \ell_{s}(m)=\mu \ell_{s}(m) \\
& =\mu(m),
\end{aligned}
$$

for all $m \in X$ and $s \in P(\varphi)$. Thus, $T_{s}(Y) \subseteq Y$, for every $s \in P(\varphi)$. Furthermore,

$$
\begin{aligned}
T_{s} T_{s^{\prime}} \nu(m) & =T_{s} \ell_{s^{\prime}}^{*} \nu(m)=\ell_{s}^{*} \ell_{s^{\prime}}^{*} \nu(m)=\ell_{s^{\prime}}^{*} \nu\left(\ell_{s} m\right) \\
& =\nu\left(\ell_{s^{\prime}} \ell_{s} m\right)=\nu\left(\ell_{s s^{\prime}} m\right)=\ell_{s s^{\prime}}^{*} \nu(m) \\
& =T_{s s^{\prime}} \nu(m),
\end{aligned}
$$

for all $\nu \in R U C(S)^{* *}, m \in R U C(S)^{*}$ and $s, s^{\prime} \in P(\varphi)$. This means that $T_{s} T_{s^{\prime}}=T_{s s^{\prime}}$, for all $s, s^{\prime} \in P(\varphi)$, i.e., $T_{s}$ is a representation on $R U C(S)^{* *}$. Since $S$ is left $\varphi$-amenable, Corollary 5.2 together with Remark 5.3 implies that there exists $\widetilde{\mu} \in Y$ such that $T_{s} \tilde{\mu}=\widetilde{\mu}$. Note that if in the definition of $T_{y}$ in the proof of Corollary 5.2, we replace $P(\varphi)$ by $S$, the relation (5.2) becomes $h\left(s y^{\prime}\right)=\varphi(s) h\left(y^{\prime}\right)$, for every $s \in S$. Thus, $s y^{\prime}=\varphi(s) y^{\prime}$, for every $s \in S$.

(ii) $\Longrightarrow$ (i) Again, consider $R U C(S)^{*}$ with the $w^{*}$-topology and suppose that the left action $\ell: S \times R U C(S)^{*} \longrightarrow R U C(S)^{*}$ is defined by $\ell_{s} m=\varphi(s) m$, for all $m \in R U C(S)^{*}$ and $s \in S$. Clearly, the map $m \longmapsto \ell_{s} m$ is continuous. Set

$$
X=\left\{m \in R U C(S)^{*}: m(\varphi)=1\right\} .
$$

Note that by Hahn-Banach Theorem the set $\left\{m \in R U C(S)^{*}: m(\varphi)=1\right\}$ is nonempty. Therefore, there exists at least one $\mathbf{m} \in X$ such that $\mathbf{m}(\varphi)=1$. Set $Y=\mathbb{C m}$. Clearly, $Y$ is $P(\varphi)$-invariant. Define $\mu$ on $Y$ by $\mu(\lambda \mathbf{m})=\lambda$, for every $\lambda \in \mathbb{C}$. Then

$$
\mu \ell_{s}(\mathbf{m})=\mu(\varphi(s) \mathbf{m})=\mu(\mathbf{m}),
$$


for all $s \in P(\varphi)$. This means that $\mu$ is $P(\varphi)$-invariant under $\ell$ and (ii) implies that any extension $\widetilde{\mu}$ is $S_{\varphi}$-invariant under $\ell$ i.e., $\widetilde{\mu} \ell_{s}=\varphi(s) \widetilde{\mu}$, for every $s \in S$. Then

$$
\widetilde{\mu} \ell_{s}(\mathbf{m})=\widetilde{\mu}(s \cdot \mathbf{m})=\widetilde{\mu}(\varphi(s) \mathbf{m}),
$$

for every $s \in S$. Hence, $s \cdot \mathbf{m}=\varphi(s) \mathbf{m}$, for every $s \in S$, because $\widetilde{\mu}$ separates points of $R U C(S)^{*}$. This implies that

$$
\mathbf{m}(f \cdot s)=s \cdot \mathbf{m}(f)=\varphi(s) \mathbf{m}(f),
$$

for every $f \in R U C(S)$ and $s \in S$. Thus, $S$ is left $\varphi$-amenable.

(i) $\Longrightarrow$ (iii) Assume that $Y$ is a closed Banach submodule of $X \in{ }_{\varphi} \mathcal{M}^{S}$, then the quotient Banach space $X / Y$ is a Banach $S$-bimodule. Set

$$
\mathcal{K}=\bigcap_{s \in S}\left\{y^{*} \in Y^{*}: s \cdot y^{*}=\varphi(s) y^{*}\right\} \text { and } \mathcal{X}=\bigcap_{s \in S}\left\{x^{*} \in X^{*}: s \cdot x^{*}=\varphi(s) x^{*}\right\} .
$$

Suppose that $\theta \in \mathcal{K}$ and $\widetilde{\theta} \in X^{*}$ is an extension of $\theta$. Then $\rho: Y^{\perp} \longrightarrow(X / Y)^{*}$ is an onto isometry and $S$-module morphism, where

$$
Y^{\perp}=\left\{x^{*} \in X^{*} \mid\left\langle y, x^{*}\right\rangle=0 \text {, for every } y \in Y\right\} .
$$

Then

$$
(s \cdot \widetilde{\theta})(y)-(\widetilde{\theta} \cdot s)(y)=(s \cdot \widetilde{\theta}-\varphi(s) \widetilde{\theta})(y)=0,
$$

for all $s \in S$ and $y \in Y$. Therefore $s \cdot \widetilde{\theta}-\varphi(s) \widetilde{\theta} \in Y^{\perp}$. Define $D: S \longrightarrow(X / Y)^{*}$ by $D(s)=\rho(s \cdot \widetilde{\theta}-\varphi(s) \widetilde{\theta})$, for every $s \in S$. Then

$$
D(s t)=\rho(s t \cdot \widetilde{\theta}-\varphi(s t) \widetilde{\theta}),
$$

for all $s, t \in S$. On the other hand,

$$
\begin{aligned}
s \cdot D(t)+D(s) \cdot t & =s \cdot \rho(t \cdot \widetilde{\theta}-\varphi(t) \widetilde{\theta})+\rho(s \cdot \widetilde{\theta}-\varphi(s) \widetilde{\theta}) \cdot t \\
& =\rho(s t \cdot \widetilde{\theta}-\varphi(s) \varphi(t) \widetilde{\theta}) \\
& =D(s t),
\end{aligned}
$$

for all $s, t \in S$. This implies that $D$ is a derivation. (i) follows that there exists $\mathfrak{y} \in(X / Y)^{*}$ such that $D(s)=s \cdot \mathfrak{y}-\mathfrak{y} \cdot s$, for every $s \in S$. Surjectivity of $\rho$ leads to there exists $\mathfrak{z} \in Y^{\perp}$ such that $D(s)=s \cdot \rho(\mathfrak{z})-\varphi(s) \rho(\mathfrak{z})$, for all $s \in S$. Now, set $\mathfrak{x}=\widetilde{\theta}-\mathfrak{z}$. Then

$$
(s \cdot(\widetilde{\theta}-\mathfrak{z})-\varphi(s)(\widetilde{\theta}-\mathfrak{z}))(y)=0,
$$

for all $s \in S$ and $y \in Y$. Thus $\mathfrak{x} \in \mathcal{X}$. This completes the proof.

(iii) $\Longrightarrow$ (iv) Consider $X^{*} \widehat{\otimes} X$ as a Banach $S$-bimodule by the following actions

$$
(f \otimes x) \cdot s=f \otimes x \cdot s \quad \text { and } \quad s \cdot(f \otimes x)=f \otimes \varphi(s) x=\varphi(s) f \otimes x,
$$

for all $s \in S, x \in X$ and $f \in X^{*}$. Set $\mathfrak{B}(X)=\{T \in \mathbf{B}(X): s \bullet T=\varphi(s) T\}$ where is the module product of $S$ on $\mathbf{B}(X)$. Consider the following sets

$$
H:=\varlimsup\left\{T^{*}(f) \otimes x-f \otimes T(x): T \in \mathfrak{B}(X), f \in X^{*}, x \in X\right\}
$$


and

$$
K:=\varlimsup\{f \otimes x: f \in \mathcal{X}\},
$$

where by $\overline{l i n}$, we mean the closed linear span. Let $Y$ be the closed linear span of $H$ and $K$. Clearly, $H$ and $K$ are Banach $S$-submodules of $X^{*} \widehat{\otimes} X$. Therefore the quotient space $Y / H$ is a Banach $S$-submodule of $\left(X^{*} \widehat{\otimes} X\right) / H$. Let $\theta \in\left(X^{*} \widehat{\otimes} X\right)^{*}$ that satisfies $\theta(f \otimes x)=f(x)$, for all $x \in X$ and $f \in X^{*}$. Then

$$
\theta\left(T^{*}(f) \otimes x-f \otimes T(x)\right)=T^{*}(f)(x)-f(T(x))=0,
$$

for all $x \in X$ and all $f \in X^{*}$. This means that $\theta \in H^{\perp}$. Pick $\vartheta \in\left(\left(X^{*} \widehat{\otimes} X\right) / H\right)^{*}$ such that $\vartheta(y+H)=\theta(y)$, for every $y \in X^{*} \widehat{\otimes} X$. By (5.4), we have

$$
\begin{aligned}
(s \cdot \vartheta-\vartheta \cdot s)(f \otimes x+H) & =\vartheta((f \otimes x+H) \cdot s)-\vartheta(s \cdot(f \otimes x+H)) \\
& =\vartheta(f \otimes x \cdot s+H)-\varphi(s) \vartheta(f \otimes x+H) \\
& =\theta(f \otimes x \cdot s)-\varphi(s) \theta(f \otimes x),
\end{aligned}
$$

for all $s \in S, x \in X$ and all $f \in X^{*}$. Since $\alpha \in H^{\perp}$, if we apply (5.6), then

$$
s \cdot \vartheta=\varphi(s) \vartheta,
$$

for all $s \in S$ and all $\vartheta \in(Y / H)^{*}$. The part (ii) implies there is an extension $\widetilde{\vartheta}$ of $\vartheta$ such that $\widetilde{\vartheta} \in(X / H)^{*}$ and $s \cdot \widetilde{\vartheta}=\varphi(s) \widetilde{\alpha}$. Define $P(f)(x)=\widetilde{\vartheta}(f \otimes x+H)$, for all $x \in X$ and $f \in X^{*}$. Clearly, $P$ is bounded. Moreover,

$$
\begin{aligned}
(P \circ P(f))(x) & =P(P(f))(x)=P(\widetilde{\vartheta}(f \otimes x+H))=P(f(x)) \\
& =P(f)(x)
\end{aligned}
$$

for all $x \in X$ and $f \in X^{*}$. This shows that $P$ is a bounded projection from $X^{*}$ onto $X^{*}$. Let $T \in \mathfrak{B}(X)$, then by (5.5), we have

$$
\begin{aligned}
P T^{*}(f)(x) & =\widetilde{\vartheta}\left(T^{*}(f) \otimes x\right)=T^{*}(f)(x) \\
& =\vartheta(f \otimes T(x))=\widetilde{\vartheta}(f \otimes T(x)+H)=P(f)(T(x)) \\
& =T^{*} P(f)(x),
\end{aligned}
$$

for all $x \in X$ and all $f \in X^{*}$. Thus, $P$ commutes with every $T^{*} \in \mathfrak{B}\left(X^{*}\right)$.

(iv) $\Longrightarrow$ (i) Assume that $X=R U C(S)^{*} \widehat{\otimes} R U C(S)^{*}$, which $X$ becomes a Banach $S$-bimodule by the following actions

$$
s \cdot(f \otimes g)=\varphi(s) f \otimes g \quad \text { and } \quad(f \otimes g) \cdot s=f \otimes g,
$$

for all $f, g \in R U C(S)^{*}$ and $s \in S$. Let $\mathcal{T}=\left\{r_{s}: s \in S\right\} \cup\left\{l_{s}: s \in S\right\}$ be a family of bounded linear operators from $X$ into $X$, such that

$$
l_{s}(f \otimes g)=\varphi(s) f \otimes g \quad \text { and } \quad r_{s}(f \otimes g)=f \otimes g,
$$

for all $f, g \in R U C(S)^{*}$ and $s \in S$. Then

$$
t \cdot l_{s}(f \otimes g)=\left(l_{s} \cdot t\right)(f \otimes g),
$$

and

$$
t \cdot r_{s}(f \otimes g)=\left(r_{s} \cdot t\right)(f \otimes g),
$$


for all $f, g \in R U C(S)^{*}$ and $s, t \in S$. Thus, every member of $\mathcal{T}$ commutes with the action of $S$ on $X$. Then (iii) implies that there is a bounded surjective projection $P: X^{*} \longrightarrow X^{*}$ such that $P T^{*}=T^{*} P$, for all $T \in \mathcal{T}$.

Define $\tau: X^{*} \longrightarrow X^{*}$ by $\tau(F)(f \otimes g)=F(g \otimes f)$, for all $F \in X^{*}$ and $f, g \in$ $R U C(S)^{*}$. Then

$$
\begin{aligned}
\tau(F \cdot s)(f \otimes g) & =(F \cdot s)(g \otimes f)=\varphi(s) F(g \otimes f) \\
& =\tau(F)(\varphi(s) f \otimes g)=\tau(F)\left(l_{s}(f \otimes g)\right) \\
& =l_{s}^{*} \tau(F)(f \otimes g)
\end{aligned}
$$

for all $F \in X^{*}, f, g \in R U C(S)^{*}$ and $s, t \in S$. Consider the projective mapping $\pi$ : $R U C(S)^{*} \widehat{\otimes} R U C(S)^{*} \longrightarrow R U C(S)^{*}$ defined by $\pi(f \otimes g)=f g$, for all $f, g \in R U C(S)^{*}$ Set $M=\tau^{*}\left(P^{*}(I \otimes I)\right)$, where $I$ is the identity function in $R U C(S)^{*}$. Since $\pi^{* *} M \in$ $R U C(S)^{* * *}$,

$$
s \cdot \pi^{* *} M=\varphi(s) \pi^{* *} M
$$

for every $s \in S$. Then by properties of $P$, we have

$$
\begin{aligned}
\pi^{* *} M(F) & =\pi^{* *} \tau^{*}\left(P^{*}(I \otimes I)\right)(F)=\left(P^{*}(I \otimes I)\right)\left(\tau \pi^{*}(F)\right) \\
& =\left(P \tau \pi^{*} F\right)(I \otimes I)=\left(\tau \pi^{*}(F)\right)(I \otimes I) \\
& =\pi^{*}(F)(I \otimes I)=F \pi^{* *}(I \otimes I) \\
& =F(I),
\end{aligned}
$$

for every $F \in R U C(S)^{* *}$. Now, we set $m=\left.\pi^{* *} M\right|_{R U C(S)}$. Then (5.12) implies that $s \cdot m=\varphi(s) m$, for every $s \in S$. Thus,

$$
s \cdot m(f)=m(f \cdot s)=\varphi(s) m(f),
$$

for every $f \in R U C(S)$ and $s \in S$. The relation (5.13) shows that, for any $f \in R U C(S)$, $\pi^{* *} M(f)=I(f)=1$. This implies that $m(\varphi)=1$ and consequently, $S$ is left $\varphi$ amenable.

For the right case, similarly, by defining the right Hahn-Banach Property, we can prove the above theorem.

\section{Acknowledgment}

The authors would like to thank the referee for careful reading of the paper and for his/her useful suggestions, which greatly improved the presentation of the paper.

\section{References}

[1] L. N. Argabright, Invariant means and fixed points; a sequel to Mitchell's papers, Trans. Amer. Math. Soc., 130(1968), 127-130.

[2] A. C. Baker and J. W. Baker, Duality of topological semigroups with involution, J. London Math. Soc., 44(1969), 251-260.

[3] T. M. Bisgaard, Extension of characters on *-semigroups, Math. Ann., 282(1988), 251-258.

[4] W. Eberlein, Abstract ergodic theorems and weak almost periodic functions, Trans. Amer. Math. Soc., 67(1949), 217-240. 
[5] H. G. Dales, Banach algebras and automatic continuity, London Math. Society Monographs, Volume 24, Clarendon Press, Oxford, 2000.

[6] H. G. Dales, A. M. Lau and D. Strauss, Banach algebras on semigroups and on their compactifications, Mem. Amer. Math. Soc., 2010.

[7] M. Day, Means for the bounded functions and ergodicity of the bounded representations on semigroups, Trans. Amer. Math. Soc., 69(2)(1950), 276-291.

[8] M. Day, Amenable semigroups, Illinois J. Math., 1(1957), 509-544.

[9] K. Fan, Invariant subspaces for a semigroup of linear operators, Indug. Math., 27(1965), 447-451.

[10] N. Grønbæk, Amenability of weighted discrete semigroup convolution algebras, Proc. Royal Soc. Edinburgh, Section A, 110(1988), 351-360.

[11] B. E. Johnson, Cohomology in Banach algebras, Mem. Amer. Math. Soc., Providence, R.I., 127(1972).

[12] E. Kaniuth, A. T. Lau and J. Pym, On $\varphi$-amenability of Banach algebras, Math. Proc. Camb. Phil. Soc., 144(2008), 85-96.

[13] M. Klawe, Semidirect product of semigroups in relation to amenability, cancelation properties, and strong Følner conditions, Pacific J. Math., 73(1)(1977), 91-106.

[14] A. M. Lau, Finite dimensional invariant subspaces for a semigroup of linear operators, J. Math. Anal. Appl., 91(1983), 314-319.

[15] M. Maysami Sadr and A. Pourabbas, Johnson amenability for topological semigroups, Iran. J. Sci. Tech. Trans. A, 32(A2)(2010), 151-160.

[16] T. Mitchell, Topological semigroups and fixed points, Illinois J. Math., 14(1970), 636-641.

[17] M. S. Monfared, Character amenability of Banach algebras, Math. Proc. Camb. Phil. Soc., 144(2008), 697-706.

[18] I. Namioka, Følners conditions for amenable semigroups, Math. Scand., 15(1964), 18-28.

[19] I. Namioka, On certain actions of semi-groups on L-spaces, Studia Math., 29(1967), 63-77.

[20] J. M. A. M. van Neerven, Hahn-Banach type theorems for adjoint semigroups, Math. Ann., 287(1990), 63-71.

[21] A. T. Paterson, Amenability, Amer. Math. Soc. Math. Survey and Monographs 29, Rhode Island: Providence, 1988.

[22] N. J. Rothman, On the uniqueness of character semigroups, Math. Ann., 151(1963), 346-354.

[23] J. R. Silverman, Means on semigroups and the Hahn-Banach extension property, Trans. Amer. Math. Soc., 83(1956), 222-237.

Young Researchers and Elite Club, Islamic Azad University, Ardabil Branch

E-mail address: jabbari_al@yahoo.com

${ }^{2}$ Department of Mathematics, Urmia University

TEHRAN, IRAN

E-mail address: ebadian.ali@gmail.com

${ }^{3}$ Department of Mathematics, Semnan University

P. O. Box 35195-363, Semnan, Iran

E-mail address: madjid.eshaghi@gmail.com 